\title{
High-efficiency x-ray gratings with asymmetric-cut multilayers
}

\author{
Saša Bajt, ${ }^{1, *}$ Henry N. Chapman,${ }^{2,3}$ Andrew Aquila, ${ }^{1,5}$ and Eric Gullikson ${ }^{4}$ \\ ${ }^{1}$ Photon Science, DESY, Notkestrasse 85, 22607 Hamburg, Germany \\ ${ }^{2}$ Center for Free-Electron Laser Science, DESY, Notkestrasse 85, 22607 Hamburg, Germany \\ ${ }^{3}$ Department of Physics, University of Hamburg, Luruper Chaussee 149, 22761 Hamburg, Germany \\ ${ }^{4}$ Advanced Light Source, Lawrence Berkeley National Laboratory, 1 Cyclotron Road, Berkeley, California 94720, USA \\ ${ }^{5}$ Current address: XFEL GmbH, Notkestrasse 85, 22607 Hamburg, Germany \\ ${ }^{*}$ Corresponding author: sasa.bajt@desy.de
}

Received August 15, 2011; revised October 28, 2011; accepted November 11, 2011;

posted November 16, 2011 (Doc. ID 152933); published February 9, 2012

\begin{abstract}
We present the fabrication and analysis of efficient and highly dispersive gratings for the x-ray and extreme ultraviolet (EUV) regime. We show that an asymmetric-cut multilayer structure can act as a near-perfect blazed grating. The precision and high line density are achieved by layer deposition of materials, which can be controlled to the angstrom level. We demonstrate this in the EUV regime with two structures made by cutting and polishing magnetron-sputtered multilayer mirrors of over 2000 bilayers thick, each with a period of $6.88 \mathrm{~nm}$. These were cut at angles of $2.9^{\circ}$ and $7.8^{\circ}$ to the surface. Within the $3 \%$ bandwidth rocking curve of the multilayer, the angular dispersion of the diffracted wave was in agreement with the grating equation for elements with 7250 and 19,700 line pairs $/ \mathrm{mm}$, respectively. The dependence of the measured efficiency was in excellent agreement with a formulation of dynamical diffraction theory for multilayered structures. At a wavelength of $13.2 \mathrm{~nm}$, the efficiency of the first-order diffraction was over $95 \%$ of the reflectivity of the uncut multilayer. We predict that such structures should also be effective at shorter x-ray wavelengths. Both the Laue (transmitting) and Bragg (reflecting) geometries are incorporated in our formalism, which is applied to the analysis of multilayer Laue lenses and focusing and dispersing Bragg optics. (c) 2012 Optical Society of America
\end{abstract}

OCIS codes: $\quad 050.0050,050.1960,050.7330,340.0340,340.7480$.

\section{INTRODUCTION}

Because of the weak refraction in all materials, optical elements for hard and soft $\mathrm{x}$ rays and extreme ultraviolet (EUV) radiation generally rely upon the process of diffraction. This requires the fabrication of structures at length scales comparable to the wavelength of the radiation. For example, the resolution of a zone plate, which is proportional to the wavelength divided by the numerical aperture of the zone plate, is equal to the smallest zone width of the element. Similarly, the dispersion of a grating is maximized by reducing the linewidth to the limit of the wavelength. However, because the interaction of $x$ rays with materials is weak, the scattering efficiency of structures approaching the size of a wavelength is low. Substantial diffraction efficiency from such small feature sizes is only possible if the radiation field interacts throughout a large volume of material. Such is the case of hard x-ray crystal diffraction, where an efficiency up to $100 \%$ can be reached. In the soft x-ray regime, artificial multilayer structures attain high reflectivity through the interaction of many layers through the depth of the stack [1,2]. Similarly, highnumerical-aperture zone plates, for example, require diffraction occurring throughout a volume to achieve high efficiency [3]. Achieving high performance therefore requires the tailoring of structures in three dimensions with a precision well below $10 \mathrm{~nm}$. The layer-by-layer deposition of materials (of thickness comparable to the wavelength) onto a substrate, followed by patterning or shaping, provides a means to achieve volume optical elements with the required precision and quality at the scale of a wavelength.
Gratings are primarily manufactured today by ruling or by other means of modifying an initially smooth surface. For the soft x-ray and EUV regime, such elements are often overcoated with a multilayer, conformal to the substrate profile, to increase the reflectivity of a particular order [늠ㅁ. The most efficient soft x-ray blazed grating substrates have been made by multiple-level electron-beam lithography [7] or wet anisotropic etching of silicon [6]. All multilayer-coated gratings suffer inefficiencies due to the unavoidable imperfections in gratings manufactured by ruling. Diffraction efficiency is reduced by deviation of the grating facets from the ideal sawtooth shape, rough facets, and the rounding of the sawtooth shape by the multilayer coating. These imperfections also cause multiple diffraction orders and ghost fringes.

In this paper we explore the fabrication and characterization of high-quality gratings for EUV and soft $\mathrm{x}$ rays by using the volume diffraction effect in asymmetrically cut layered structures. The high quality is obtained by the angstromprecision of material deposition by sputtering which reveals a grating structure when sliced at an angle. $\mathrm{X}$ rays reflect from the multilayer structure, yet they are angularly dispersed due to the asymmetric truncation of the stack. We explain how such a structure acts as a blazed grating, free of imperfections of ruled gratings, and show that optics of extremely high dispersion can indeed be fabricated by layer deposition followed by asymmetric slicing, by measuring the EUV diffraction from blazed gratings with almost 20,000 line pairs per millimeter.

We first describe diffraction from asymmetric-cut multilayers in Section $\underline{2}$ and model the volume diffraction with 
dynamical diffraction theory in Section 3 . The fabrication of these elements is described and discussed in Section 4. Measurements of reflectivity and dispersion of these components are presented in Section 5. These measurements are compared with calculations using the formalism and conventions we relate in Section 3. We find excellent agreement between the theory and experiment, which indicates that these structures do indeed approach the ideal in terms of their function. Finally, in Section 6, different applications of these novel optical elements are presented, including their use as monochromators, spectrometers, pulse compressors, and volume zone plates.

\section{ASYMMETRIC-CUT MULTILAYERS}

The highest efficiency in a diffracted order of a plane grating is achieved by blazing. The blaze condition is satisfied when the diffracted light of the particular order is specularly reflected from the groove facets. That is, for incident and diffracted angles of $\alpha$ and $\beta$, respectively, as shown in Fig. 1 , the blaze angle should satisfy $2 \phi=\alpha+\beta$. In this case, the grating equation is

$$
\begin{aligned}
n \lambda & =D(\sin \alpha-\sin \beta), \\
& =2 D \cos \phi \sin \theta,
\end{aligned}
$$

where $D$ is the grating period, $\theta$ is the grazing angle of specular reflection from the groove facets $(\theta=\alpha-\phi=\phi-\beta)$, and $n$ is the grating diffracted order.

In the EUV-to-X-ray wavelength region, the efficiency of both laminar and blazed gratings is much enhanced by coating the grating with a multilayer coating []․ Ignoring a small correction due to refraction in the coating, the Bragg condition for which efficient reflection occurs is given by

$$
m \lambda=2 d \sin \theta_{B}
$$

where $d$ is the multilayer period, $m$ is the diffraction order, and $\theta_{B}$ is the same angle of reflection from the grating facets, as given above. It is clear then, by comparing Eqs. (2) and (ㅁ)

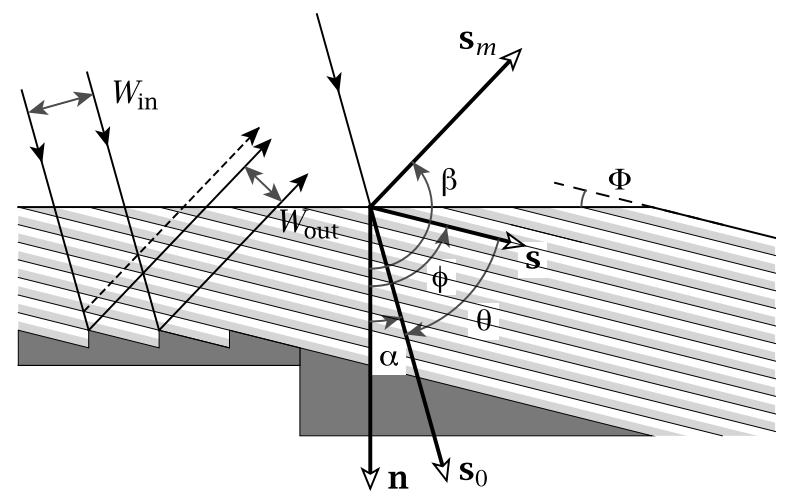

Fig. 1. A multilayer is cut at an angle $\Phi=\pi / 2-\phi$ (right). The reflection from the asymmetric-cut multilayer film (center) is identical to reflection from an ideal multilayer-coated blazed grating (left) [5] because the multilayer structure is the same in both cases. The incident wave is in the direction $\mathbf{s}_{0}$ and the reflected wave is in the direction $\mathbf{s}_{m}$. The sense of the angles and the direction of the trace of the surfaces $s$ is defined by $0<\theta_{B}=\left(\mathbf{s}, \mathbf{s}_{0}\right)<\pi / 2$, and so the angles $\alpha=\left(\mathbf{n}, \mathbf{s}_{0}\right)$, $\beta=\left(\mathbf{n}, \mathbf{s}_{m}\right), \phi=(\mathbf{n}, \mathbf{s})$ are all negative in this diagram. A beam of width $W_{\text {in }}$ exits with width $W_{\text {out }}$. that, when the first-order blaze and Bragg conditions are simultaneously met, the step height of the facets, given by $D \cos \phi$, is equal to the multilayer period, $d$. That is, the layers of the multilayer coating are in phase with the facets of the grating: the top of the first layer period deposited above a particular facet of the grating will be in line with the adjacent facet. In general, the $n$th grating order can be matched to the $m$ th-order reflection from the multilayer, in which case $(D / n) \cos \phi=d / m$ [5].

The above discussion immediately suggests that a blazed grating can be made quite simply by cutting, or slicing a multilayer coating so that the surface normal is at an angle $\phi$ to the layers rather than the usual $90^{\circ}$. The Bragg-reflected light from such an asymmetric-cut multilayer will behave just as light diffracting from a blazed grating with period $D=d /(m \cos \phi)$. A sliced multilayer should be indistinguishable from a perfect blazed grating that is multilayer coated, as long as the number of multilayer periods is larger than the extinction depth. The only difference between the two cases is that the top surface of the sliced multilayer is flat, whereas the top surface of a multilayer-coated grating will (ideally) conform to the blazed grating substrate. However, because in both cases the reflection is a volume effect and arises from the addition of reflected fields from every layer interface, the small missing material at the top will only cause a small refractive change (the difference between a triangular cross-section of vacuum or multilayer material). The equivalence between a grating and an asymmetric multilayer has been noted before [른 $\underline{4}$, demonstrated, and applied [욤] . This equivalence is also valid in the case of diffraction from crystals, where it is well known that the diffracted $\mathrm{x}$ rays from an asymmetric-cut crystal are dispersed, in accordance with the grating equation $[10,11]$. In fact, dynamical diffraction theory can be applied with full validity to the case of asymmetric-cut multilayers, as is presented in Section 3 .

Thick multilayers can also be used in the transmitting Laue geometry, and in this regard they are analogous to a perfect blazed transmission grating, based on refraction. In this case the analogous transmission grating might be physically impossible due to the limited change in refractive index that can be achieved. For example, symmetric Laue diffraction is analogous to a double-sided refractive blazed grating. However, unlike the blazed reflection grating, adding a multilayer to the surface of the transmission grating does not produce the analogous Laue structure.

To describe a completely general geometry we follow the sign conventions of angles as used by Authier [12]. As depicted in Fig. 1, the normal unit vector, directed into the surface, is given by $\mathbf{n}$, the direction of the incident beam is given by the unit vector $\mathbf{s}_{0}$ and that of the diffracted beam is given by $\mathbf{s}_{m}$. The trace of the layers is given by $\mathbf{s}$. The quantities $\alpha, \beta$, and $\phi$ are the angles between $\mathbf{n}$ and the incident, reflected, and facet directions, respectively: $\alpha=\left(\mathbf{n}, \mathbf{s}_{0}\right), \beta=\left(\mathbf{n}, \mathbf{s}_{m}\right), \phi=$ $(\mathbf{n}, \mathbf{s})$. The sense of the angles is set by defining $\left(\mathbf{s}, \mathbf{s}_{0}\right) \equiv$ $+\theta_{B}$ at the Bragg condition, where $0 \leq \theta_{B}<\pi / 2$ for a positive diffraction order. For Bragg reflection, we then have the following relationships:

$$
-\frac{\pi}{2}<\alpha<-\frac{\pi}{2}+2 \theta_{B}
$$




$$
\begin{gathered}
-\frac{\pi}{2}-2 \theta_{B}<\beta<-\frac{\pi}{2}, \\
-\frac{\pi}{2}-\theta_{B}<\phi<-\frac{\pi}{2}+\theta_{B} .
\end{gathered}
$$

Just as for an asymmetric-cut crystal, diffraction from an asymmetric-cut multilayer in the Bragg or Laue geometry can be described an asymmetry parameter [13], $b$, given by

$$
b=\frac{\mathbf{n} \cdot \mathbf{s}_{0}}{\mathbf{n} \cdot \mathbf{s}_{m}}=\frac{\gamma_{0}}{\gamma_{m}}=\frac{\cos \alpha}{\cos \beta}
$$

where the direction cosines $\gamma_{0}=\mathbf{n} \cdot \mathbf{s}_{o}$ and $\gamma_{m}=\mathbf{n} \cdot \mathbf{s}_{m}$. Note that

$$
|b|=\frac{W_{\text {in }}}{W_{\text {out }}}
$$

is the ratio of the widths of the incident and diffracted beams. The asymmetry parameter can also be applied to any order of diffraction from a grating (blazed or not). In the x-ray crystal diffraction literature, the asymmetry parameter is sometimes defined as the inverse of Eq. (7). We follow the convention used in $[11,13]$. For the Bragg geometry, where the diffracted light exits on the same side of the surface as the incident beam, we have $b<0$, whereas $b>0$ describes Laue diffraction. Specifically, $b=-1$ corresponds to symmetric Bragg diffraction and $b=1$ corresponds to symmetric Laue diffraction. Note also from Eq. (1) we have

$$
\frac{\partial \beta}{\partial \alpha}=b
$$

The properties of asymmetric multilayers are naturally described in terms of the asymmetry parameter $b$ (the geometry of the reflection) and the Bragg angle $\theta_{B}$ (the period of the multilayer). Often in experimental design we wish to determine the cut angle $\phi$ that satisfies a given $b$ and $\theta_{B}$, which can be found by solving

$$
b=\frac{\cos \left(\phi+\theta_{B}\right)}{\cos \left(\phi-\theta_{B}\right)} .
$$

This equation has the four solutions given by

$$
\phi= \pm \cos ^{-1}\left[ \pm \frac{(1+b) \sin \theta_{B}}{\sqrt{1+b^{2}-2 b \cos 2 \theta_{B}}}\right] \text {. }
$$

In the case of Bragg reflection, we must have $-\pi<\phi<0$, from Eq. (6). When $-1<b<0$, which is the beam expanding geometry for which $-\pi<\phi<-\pi / 2$, we have $1+b$ positive and so

$$
\begin{aligned}
\phi & =-\cos ^{-1}\left[-\frac{(1+b) \sin \theta_{B}}{\sqrt{1+b^{2}-2 b \cos 2 \theta_{B}}}\right] \\
& =-\cos ^{-1}\left[-\frac{m(1+b) \lambda}{2 \sqrt{(b-1)^{2} d^{2}+m^{2} \lambda^{2} b}}\right],
\end{aligned}
$$

where the usual definition of $\cos ^{-1}$ is used, which has a range of $[0, \pi)$. In the beam condensing Bragg geometry, for which $-\pi / 2<\phi<0$ and $1+b$ is negative, Eq. (12) is also the correct solution. A similar analysis can be carried out in the Laue geometry, where $b>0$. In this case, however, we can have $-\pi / 2<\phi<\pi / 2$, for which $\cos \phi>0$. In Laue geometry, when $-\pi / 2<\phi<0$, we always have the incident beam at a shallower angle from the surface than the transmitted, corresponding to $b>1$. Thus, in general for Bragg and Laue geometries, the solutions to Eq. (10) are

$$
\phi=-\cos ^{-1}\left[-\frac{(1+b) \sin \theta_{B}}{\sqrt{1+b^{2}-2 b \cos 2 \theta_{B}}}\right], \quad b<0,
$$

$$
\phi=\cos ^{-1}\left[\frac{(1+b) \sin \theta_{B}}{\sqrt{1+b^{2}-2 b \cos 2 \theta_{B}}}\right], \quad 0<b<1,
$$

$$
\phi=-\cos ^{-1}\left[\frac{(1+b) \sin \theta_{B}}{\sqrt{1+b^{2}-2 b \cos 2 \theta_{B}}}\right], \quad b>1 .
$$

The dispersion of the diffracted light from an asymmetriccut multilayer follows directly from the grating equation (Eq. 1), from which

$$
\frac{\partial \beta}{\partial \lambda}=-\frac{1}{\lambda}(1+b) \tan \theta_{B}
$$

As expected, there is no dispersion for symmetric Bragg geometry $(b=-1)$ because there is no grating structure in that case. The dispersion is negative for expanding Bragg reflections, $-1<b<0$, and positive for condensing Bragg reflections, $b<-1$. Equation (16) is also valid for Laue diffraction. The dependence of the angular dispersion on the multilayer period is implicit through the Bragg angle $\theta_{B}$, defined by the Bragg equation (Eq. 3), and the dependence on the incidence and exit angles $\alpha$ and $\beta$ is implicit through the asymmetry parameter $b$. These angles can be determined from the cut angle $\phi$, as given above, and noting that $\alpha=\phi+\theta_{B}$ and $\beta=\phi-\theta_{B}$. In the reflection geometry, the largest dispersion for a given multilayer period occurs for $b \rightarrow \infty$, which corresponds to grazing exit angle $(|\beta| \rightarrow \pi / 2)$ where the beam width is condensed on reflection. As has been pointed out elsewhere, this is a consequence of Liouville's theorem. A quasi-monochromatic collimated beam (of finite width) that is reduced in width must be dispersed in angle to preserve the space-angle phase volume.

\section{DYNAMICAL DIFFRACTION OF ASYMMETRIC MULTILAYERS}

The optical properties of asymmetric-cut multilayers can be calculated by solving Maxwell's equations for fields interacting with the periodic structure (through the susceptibility of the material), with the application of the appropriate boundary conditions, and with the ersatz solution of a sum of an incident and diffracted plane wave. Such a system has been solved for this case of sliced multilayers [8], but we note that the problem is exactly that addressed by the dynamical diffraction theory of crystals for which a huge body of literature exists. In the analysis that follows, we prefer to apply the dynamical diffraction theory, because we can easily examine 
effects that are known to practitioners of crystal diffraction. Another theory that has been applied to asymmetric multilayer diffraction is coupled-wave theory $[14,15]$.

The dynamical diffraction theory gives the intensities of the diffracted and refracted beams for a slab of thickness $t$ of a material with periodic structure of period $d$. The direction of the periodicity relative to the two parallel surfaces of the slab is specified by the asymmetry parameter $b$ and the direction of the diffracted beam, $m$ [corresponding to a momentum transfer $\left.q=(4 \pi / \lambda) \sin \theta_{B}=2 \pi m / d\right]$. In the case where the susceptibilities of the layered materials are complex (the materials are absorbing as well as refractive), the intensity of the diffracted beam in the Bragg geometry is given by $\{$ see Eq. (7.11) in [12] $\}$

$$
R_{B}=\left|\frac{\chi_{m}}{\chi_{\bar{m}}}\right| \frac{\left|\epsilon_{1}-\epsilon_{2}\right|^{2}}{\left|\eta\left(\epsilon_{1}-\epsilon_{2}\right)+\sqrt{\eta^{2}-1}\left(\epsilon_{1}+\epsilon_{2}\right)\right|^{2}},
$$

with $\quad \epsilon_{1}=\exp (i \pi t / \Lambda), \quad \epsilon_{2}=\exp (-i \pi t / \Lambda), \quad$ where $\Lambda=\Lambda_{0} /\left(\eta^{2}-1\right)^{1 / 2}$. In the above,

$$
\eta=\left(\Delta \theta-\Delta \theta_{s}\right) / \delta_{s}
$$

is the deviation parameter, where $\Delta \theta=\theta-\theta_{B}$ is the departure of the incidence angle from the Bragg angle defined by Eq. (3), and

$$
\begin{gathered}
\Delta \theta_{s}=-\frac{\chi_{0}(1-1 / b)}{2 \sin 2 \theta_{B}}, \\
\delta_{s}=\frac{C \sqrt{\chi_{m} \chi_{\bar{m}}}}{\sqrt{|b|} \sin 2 \theta_{B}}, \\
\Lambda_{0}=\frac{\lambda\left|\gamma_{m}\right|}{\delta_{s} \sin 2 \theta_{B}} .
\end{gathered}
$$

The real part of $\Delta \theta_{s}$ is the deviation from Bragg's equation due to refraction, the real part of $2 \delta_{s}$ is the Darwin width or the angular width of the Bragg reflection, and the real part of $\Lambda_{0}$ is the extinction distance (at the middle of the Bragg peak, corresponding to $\eta=0$, the $1 / e$ penetration depth is $\Lambda_{0} / 2 \pi$ ). The factor $C$ depends on the polarization state of the beams: $C=1$ for $\sigma$ polarization, and $C=\cos 2 \theta_{B}$ for $\pi$ polarization. Note the inverse relationship between the Darwin width and the extinction length: the larger the extinction depth the more layers contributing to the reflection and the narrower the bandwidth of the reflection. The extinction depth is a property of the multilayer materials and is independent of the cut geometry.

Experimental characterization of soft-x-ray and EUV multilayers is often performed at fixed geometry and varying wavelength. From Eq. (3) we can formulate the equations for the reflected intensity in terms of the change in wavelength from the Bragg wavelength by substituting for the dependent parameter

$$
\Delta \theta=-\Delta \lambda / \lambda \tan \theta_{B}=-\frac{m \Delta \lambda}{2 \sqrt{d^{2}-m^{2} \lambda^{2}}}
$$

We then obtain the wavelength refractive shift $\Delta \lambda_{s}$ and Darwin width $\Delta \lambda_{w}$, relative to the Bragg wavelength $\lambda_{B}=$
$2 d \sin \theta_{B}$ and for fixed incident angle, from Eqs. (모), (20), and (22), as

$$
\begin{gathered}
\Delta \lambda_{s} / \lambda=\frac{\chi_{0}(1-1 / b)}{4 \sin ^{2} \theta_{B}}, \\
\Delta \lambda_{w} / \lambda=-\frac{C \sqrt{\chi_{m} \chi_{\bar{m}}}}{\sqrt{|b|} \sin ^{2} \theta_{B}} .
\end{gathered}
$$

In the Laue geometry, the reflected and refracted beams transmitted through a slab of thickness $t$ and asymmetry parameter $b$, are given by \{see Eq. (6.16) in [12] \}

$$
R_{L}=\left|\frac{\chi_{m}}{\chi_{\bar{m}}}\right| \exp \left[-\frac{\mu_{0}}{2}\left(\frac{1}{\gamma_{0}}+\frac{1}{\gamma_{m}}\right) t\right] \frac{\left|\epsilon_{1}-\epsilon_{2}\right|^{2}}{4\left|1+\eta^{2}\right|},
$$

$T_{L}=\exp \left[-\frac{\mu_{0}}{2}\left(\frac{1}{\gamma_{0}}+\frac{1}{\gamma_{m}}\right) t\right] \frac{\eta \eta\left(\epsilon_{1}-\epsilon_{2}\right)+\left.\sqrt{1+\eta^{2}}\left(\epsilon_{1}+\epsilon_{2}\right)\right|^{2}}{4\left|1+\eta^{2}\right|}$,

where $\mu_{0}=-2 \pi \operatorname{Im}\left\{\chi_{0}\right\} / \lambda$ is the mean linear absorption coefficient of the materials of the structure. The dimensionless parameters $\epsilon_{1}$ and $\epsilon_{2}$ are as defined previously, except that in the Laue geometry we have $\Lambda=\Lambda_{0} /\left(\eta^{2}+1\right)^{1 / 2}$. In this case the length $\Lambda_{0}$ is known as the Pendellösung distance, which is equal to the period of the oscillation of energy in the reflected and refracted beams as a function of thickness $t$. When there is no absorption, the intensity at $\Delta \theta=0$ in the reflected and refracted beams is unity and zero, respectively, for thicknesses of integer multiples of $\Lambda_{0}$.

To apply the conventional crystal dynamical diffraction theory, the structure factors of atomic unit cells of a crystal are replaced by the structure factors of the material layers. Following the usual treatment (see, for example, Subsection 2.7 in [12]),

$$
\begin{aligned}
& \chi_{m}=\frac{1}{V} \int \chi(\mathbf{r}) \exp \left(-i \mathbf{q}_{m} \cdot \mathbf{r}\right) \mathrm{d} \mathbf{r}, \\
& =\frac{1}{d} \int \chi(z) \exp (-2 \pi i m z / d) \mathrm{d} z,
\end{aligned}
$$

where $V$ is the volume of material, and we have assumed a layered structure of period $d$ and that our diffracted order $m$ satisfies the Bragg condition such that $\left|q_{m}\right|=4 \pi \sin \theta_{B} / \lambda$ $=2 \pi m / d$. The complex real-space susceptibility $\chi(z)$ can be described by the complex refractive index of the materials (neglecting any crystalline structure of the layer materials themselves). Because the refractive index is given by $n(z)=[1+\chi(z)]^{1 / 2}$, and $n(z)=1-\delta(z)-i \beta(z)$ defines the refractive index decrement $\delta$ and absorption term $\beta$, we have

$$
\chi(z) \approx-2(\delta(z)+i \beta(z))
$$

We use a layer model of two materials with sharp interfaces,

$$
\delta(z)= \begin{cases}\delta_{1}, & 0<z \leq \Gamma d \\ \delta_{2}, & \Gamma d<z \leq d\end{cases}
$$


where $\Gamma$ is the ratio between the layer thickness of layer 1 to the period and $z$ is distance into the multilayer. A similar expression holds for $\beta$. It is easily verified that with this layer model, Eq. (27) leads to

$$
\begin{gathered}
\chi_{0}=-2(\bar{\delta}+i \bar{\beta}), \\
\sqrt{\chi_{m} \chi_{\bar{m}}}=2\left(\delta_{1}-\delta_{2}+i\left(\beta_{1}-\beta_{2}\right)\right) \frac{\sin (m \pi \Gamma)}{m \pi}, \\
\left|\frac{\chi_{m}}{\chi_{\bar{m}}}\right|=1 .
\end{gathered}
$$

Here, $\bar{\delta}=\Gamma \delta_{1}+(1-\Gamma) \delta_{2}$ is the mean index decrement of the materials, and similarly for $\bar{\beta}$. The linear absorption coefficient required for the reflectivity expressions in the Laue geometry, is given by $\mu_{0}=\pi \bar{\beta} / \lambda$. Structures with more than two layers per period can be considered similarly.

Note that more realistic models of the layer materials that take into account layer roughness or interdiffusion can be applied, either by deriving $\chi_{m}$ from an analytic description of $\chi(z)$, or by applying a discrete Fourier transform on a numerical representation of the layer properties. We can represent interfaces that have diffused into each other, for example, by a refractive index profile function that varies across the interfaces according to [ㅌ]

$$
\delta(z)=\delta_{2}+\frac{1}{2}\left(\delta_{1}-\delta_{2}\right)\left[\operatorname{Erf}\left(\frac{z}{\sigma_{1}}\right)-\operatorname{Erf}\left(\frac{z-\Gamma d}{\sigma_{2}}\right)\right]
$$

and equivalently for $\beta(z)$, where $2 \sigma_{1}$ and $2 \sigma_{2}$ are the widths of the interfaces. It can be shown in this case that for $\sigma_{1}$ and $\sigma_{2} \ll$ $d$ that

$$
\begin{aligned}
\sqrt{\chi_{m} \chi_{\bar{m}}} \approx & 2\left(\delta_{1}-\delta_{2}\right. \\
& \left.+i\left(\beta_{1}-\beta_{2}\right)\right) \frac{\sin (m \pi \Gamma)}{m \pi} \exp \left(-\frac{m^{2} \pi^{2}\left(\sigma_{1}^{2}+\sigma_{2}^{2}\right)}{d^{2}}\right),
\end{aligned}
$$

and $\chi_{0}$ remains unchanged from Eq. (31).

A limitation of the framework of the dynamical diffraction theory used here is that it does require strict periodicity. Another formulation of a dynamical diffraction theory is the optical-matrix theory [17-20], in which a recursion relationship is used to find the superposition of transmitted and reflected waves at each boundary. This theory has the advantage that arbitrary nonperiodic layered structures can be analyzed [20] but is only valid in the symmetric Bragg geometry where the layers are parallel to the surface. We can therefore compare the results of this theory in this case to check our implementation of the dynamical diffraction, but it cannot be used to examine the properties of sliced multilayers. In Fig. 2 we show the symmetric Bragg $(b=-1)$ reflectivity of a Mo-Si EUV multilayer, as calculated from Eq. 17 and as calculated using the IMD program [20]. In both cases a periodic $(d=7.5 \mathrm{~nm})$ structure of layers of $4.5 \mathrm{~nm}$ thick Mo and $3.0 \mathrm{~nm}$ thick Si was simulated, with perfect interfaces, and $\sigma$-polarized EUV radiation of $13.4 \mathrm{~nm}$ wavelength. The same optical constants were used in both cases: $\delta_{\mathrm{Mo}}=0.07656, \beta_{\mathrm{Mo}}=0.0061289$, $\delta_{\mathrm{Si}}=-0.0001196, \beta_{\mathrm{Si}}=0.00181825[\underline{21}]$.
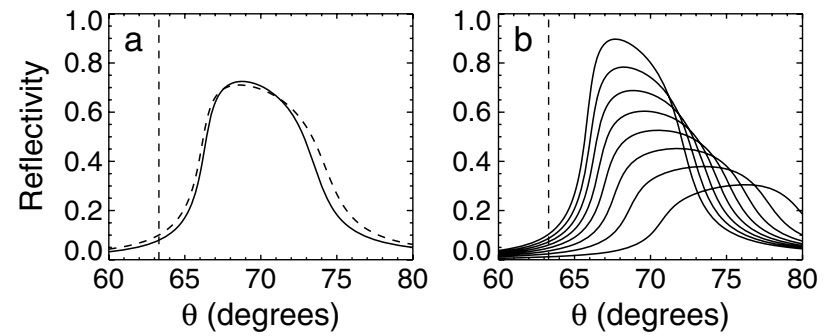

Fig. 2. a, Reflectivity at a wavelength of $13.4 \mathrm{~nm}$ for $\sigma$ polarization of a symmetric Mo-Si EUV multilayer ( $b=-1, d=7.5 \mathrm{~nm})$, as calculated by Eq. (17) with $\theta=\theta_{B}+\Delta \theta$ (solid line) and as calculated by the IMD program [20] for a multilayer with sharp interfaces (dashed line). b, $\sigma$ polarization reflectivity of the same Mo-Si structure with an asymmetric-cut angle $\phi$ varying from $-70^{\circ}$ (highest peak reflectivity) to $-140^{\circ}$ (lowest peak reflectivity), in $10^{\circ}$ steps. The Bragg angle of $63.6^{\circ}$ is indicated in panels a and $\mathrm{b}$.

There is some discrepancy between the exact calculation made by the IMD program and the result of Eq. (17), especially for $\theta>70^{\circ}$. We find that this difference is not entirely due to the approximations in the classical dynamical diffraction formalism that break down for large $\Delta \theta$, because we get essentially the same computed result when we apply the universal computational method of Huang and Dudley [22] and De Caro and Tapfer [23]. This method solves the quartic dispersion equation exactly, and it is thus applicable over full range of $\theta$. Similarly, the two-beam approximation of dynamical diffraction cannot explain the difference, for when we model a structure with a sinusoidal depth variation in the refractive index using the IMD program, we obtain less than a $0.1 \%$ change in reflectivity. The discrepancy may be due to the difference in the way the interaction between the layered structure and the electromagnetic field is taken into account in the two theories, and we note that making a change in the value of $\delta_{\text {Mo }}$ by $10 \%$ in the dynamical diffraction theory substantially reduces the discrepancy between the two models.

In Fig. $2 \mathrm{~b}$ we show computed reflectivities for the same $d=$ $7.5 \mathrm{~nm} \mathrm{E} \overline{\mathrm{UV}}$ multilayer, with various asymmetric-cut angles $\phi$, ranging from $-70^{\circ}$ to $-140^{\circ}$. From Eq. (10) $b$ varies from -1.45 to -0.25 for $\theta_{B}=63.6^{\circ}$, and as expected from Eqs. (20) and (19) the width of the reflectivity curve and the deviation from the Bragg angle increase with this progression. Note that the plots in Fig. $2 \mathrm{~b}$ show the reflectance as a function of $\theta$, achieved by varying the incidence angle $\alpha$ and essentially capturing all reflected light with a detector with a large angular acceptance. That is, these curves plot the reflectance, irrespective of the exit angle $\beta$, and do not depend on the effect of dispersion or how $\beta$ varies with $\alpha$. If we describe the experimental geometry by the angle $\Theta=\alpha-\beta$, which is the angle between the reflected and incident beams, then as the multilayer is rocked, the reflection angle will vary according to $\partial \Theta / \partial \alpha=1-b$. For symmetric reflection with $b=-1$ we get the familiar $\alpha-2 \alpha$ scans to measure multilayer reflectivity, but for the condensing geometry with $b=-2$, for example, the detector must be scanned at three times the rate of the multilayer rocking angle.

\section{FABRICATION OF SLICED MULTILAYERS}

\section{A. Low-Stress Thick Multilayer Mirrors}

We made sliced multilayers by first fabricating a Mo:Si multilayer coating on a Si wafer substrate. A coating with 2020 
periods of $3.47 \mathrm{~nm}$ thick layers of $\mathrm{Mo}$ and $\mathrm{Si}$ (period $d=6.88 \mathrm{~nm}$, and ratio of the Mo thickness to period $\Gamma=0.5)$ was made by DC magnetron sputtering [24]. This $17.5 \mu \mathrm{m}$ thick multilayer is more than 20 times thicker than required for full beam extinction for the symmetric geometry at a wavelength of $\lambda=13.2 \mathrm{~nm}$. The key to manufacturing such thick multilayer structures is to minimize the stress of the coating and to reduce the buildup of roughness in the layers. For a film of thickness $t$ and intrinsic stress $\sigma$ adhered to a thick substrate, the film tension, proportional to $\sigma t$, may deform the substrate or cause the film to de-adhere from the substrate. In conventional multilayer films of less than $100 \mathrm{bi}$ layers, developed for $13.4 \mathrm{~nm}$ EUV lithography, the film stress is usually less than $300 \mathrm{MPa}$. Increasing the thickness of this conventional multilayer by more than a factor of 50 may result in effects such as film crinkling, cracking, and de-adhesion. The inherent stress in a multilayer may be reduced by modifying the thickness ratio $\Gamma$ of the multilayer, by modifying the growth conditions of the layers with a residual gas, or by modifying the properties of the interfaces between the layers. Stress can sometimes be lowered by in situ annealing. We achieved a low stress of $<30 \mathrm{MPa}$ by choosing $\Gamma=0.5$. The RMS roughness of the 2020-period multilayer was $0.42 \mathrm{~nm}$, as measured by atomic force microscope (AFM) over spatial periods between $1.9 \mathrm{~nm}$ to $1 \mu \mathrm{m}$. This compares with the typical roughness of $0.13 \mathrm{~nm}$ for a 60 -period multilayer made for EUV lithography [25].

An additional requirement of the deposition system is that the deposition rate must not drift over the $\approx 48 \mathrm{~h}$ it takes to deposit 2020 layers. In our system, no feedback is applied to the deposition rate to control layer thickness. Instead, we rely solely upon the stability of the power supplies to achieve less than $0.1 \%$ variation of period throughout the stack. This stability was verified from x-ray reflectometry measurements made from a series of multilayer films that were fabricated before and after growing the 2020-period multilayer. The standard deviation of the measured wavelengths at peak reflectivity for this series was $0.008 \mathrm{~nm}$. Cross-section TEM imaging of the top and bottom of the 2020-period stack also showed no observable variation in period, although the precision of this measurement was about $0.07 \mathrm{~nm}$.

The structure of the uncut multilayer was probed by measuring the reflectivity as a function of wavelength, at a Bragg angle of $\theta=80^{\circ}$. The multilayer materials and thicknesses were optimized for near-normal-incidence reflection of $13.2 \mathrm{~nm}$ light, as required for EUV lithography [26], and these thick multilayers exhibit high reflectance in this wavelength region. The reflectivity is shown in Fig. 3 (dashed line), as measured at the reflectometer at beamline $\overline{6} .3 .1$ at the Advanced Light Source [27]. This measurement is sensitive to no more than the topmost 70 periods, due to extinction. The measurement confirms the $6.88 \mathrm{~nm}$ period, although the reflectivity is lower than the $67 \%$ routinely achieved on 70 -period multilayers, presumably due to the increase in interfacial roughness and interdiffusion that has occurred in this thicker structure. In this structure the diffusion occurred to such an extent that the model of Eq. (34) does not provide a good fit to the observed reflectivity. Instead, we employed a four-layer model for each period, where layers of $\mathrm{MoSi}_{2}$ (with a density of $6.13 \mathrm{~g} \mathrm{~cm}^{-3}$ ) are placed between the Si and Mo layers. The layer thicknesses found by fitting the reflectivity curve were
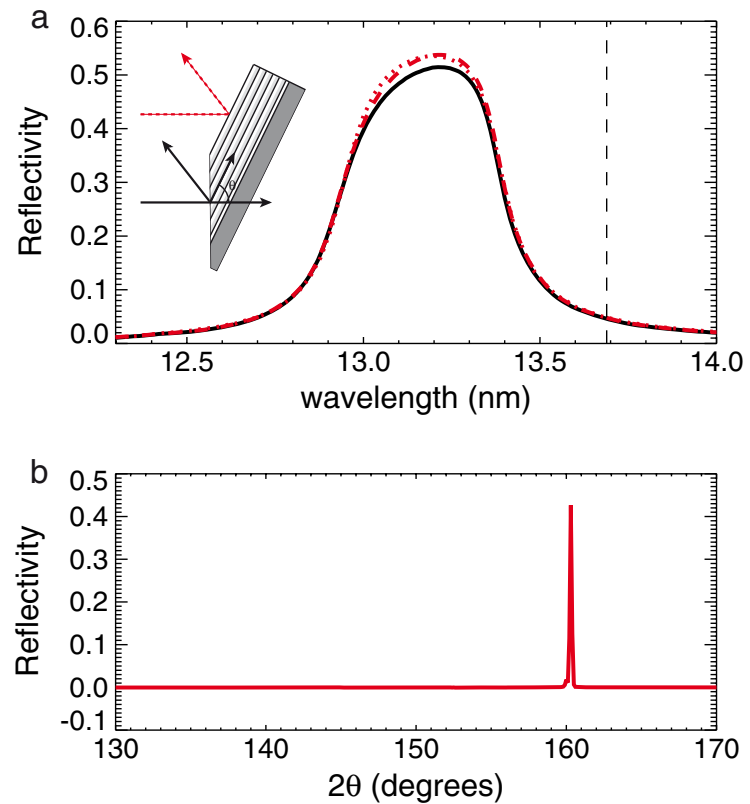

Fig. 3. (Color online) a, Measured reflectivity of the cut multilayer with $\Phi=7.8^{\circ}, \theta=80^{\circ}, \alpha=-2.2^{\circ}, \beta=-162.2^{\circ}$ (solid line) and the uncut multilayer (dashed line). The dotted line shows the calculated reflectivity of the uncut multilayer, obtained by fitting a four-layer model of the periodic structure. The wavelength satisfying the Bragg condition, Eq. (3), is indicated, and the insert depicts the experimental geo-

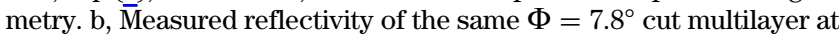
a fixed wavelength of $13.2 \mathrm{~nm}$, as a function of detector angle, $2 \theta$, for an incidence angle of $\alpha=-2.2^{\circ}$. An equivalent grating with a period $D=d / \cos \phi=50.7 \mathrm{~nm}$ would have a second-order diffraction peak at $2 \theta=143.8^{\circ}$ [as per Eq. (1)].

Si:Mo : $\mathrm{Si}_{2}: M o: M o: \mathrm{Si}_{2}=1.58 \mathrm{~nm}: 1.36 \mathrm{~nm}: 3.00 \mathrm{~nm}: 1.03 \mathrm{~nm}$, in order increasing toward the surface. Our fit includes interdiffusion parameters [see Eq. (34)] between each of these four layers of $\sigma=0.4 \mathrm{~nm}$, which agrees well with the AFM measurement of the top surface of the uncut multilayer of $0.42 \mathrm{~nm}$ RMS. In addition, we model the surface of the structure as a $5.4 \mathrm{~nm}$ layer of $\mathrm{MoSi}_{2}$ terminated with $4.8 \mathrm{~nm}$ of $\mathrm{SiO}_{2}$. The calculated reflectivity from this model is shown in Fig. 3 as a dotted line, and is seen to agree with the measured reflectivity of the uncut portion of the multilayer (dashed line).

The diffusion of the materials to form layers of $\mathrm{MoSi}_{2}$ is most probably caused by the excessive heating of the structure and substrate that occurs during the long deposition process. Usual deposition runs of 70 periods take $2-3 \mathrm{~h}$, during which time the substrate rises in temperature by less than $80^{\circ} \mathrm{C}$. During the $48 \mathrm{~h}$ deposition run for this 2020-period structure, the temperature was estimated to rise to $150^{\circ} \mathrm{C}$, which is enough to cause this diffusion-driven silicide formation [28]. As seen from our calculations, this silicide reduces the refractive index contrast and is responsible for the measured peak reflectivity of $53.7 \%$, as compared with $67 \%$ for thin Mo:Si multilayers. We expect that we could produce thick multilayers of reflectivity higher than $60 \%$ by the use of diffusion barriers $[29,30]$, combinations of materials that are more thermally stable [31] , or by cooling the substrate during deposition.

\section{B. Sliced Multilayers}

The silicon wafer with the 2020-period multilayer was cleaved into several pieces of a size about $1 \mathrm{~cm} \times 1 \mathrm{~cm}$. Asymmetric cuts to these pieces were made with a diamond saw, followed 
by polishing the resulting beveled edge with diamond paste (carried out by Evans Analytical Group, LLC, USA). The process was similar to that used to prepare thin sections of samples for imaging in a transmission electron microscope. Atomic force micrographs of two samples are shown in Fig. $\underline{4}$, for cuts made at a shallow (a) and steeper (b) angle. The cut angles were determined from EUV dispersion measurements (see Subsection 5.B) to be $\Phi=2.9^{\circ}$ and $7.8^{\circ}$, as defined in Fig. 1. These designations are confirmed by examination of the AFM height maps. Ideally, the surfaces of the polished multilayers would be plane, so that these images would be featureless, but in fact the layered structure can be seen. This may be due to a difference in polishing or oxidation rates between Mo and Si. For the sample in Fig. 4a, the period is $D=137 \mathrm{~nm}$, which agrees with $d=D \sin \bar{\Phi}$ for the known multilayer period $d=6.88 \mathrm{~nm}$. This is equivalent to a grating with 7250 line pairs $/ \mathrm{mm}$. The average step height between the exposed layers at the surface is $4.5 \mathrm{~nm}$, which is close to the thickness of the Mo layer with its silicide. Surface analysis reveals that $\mathrm{Si}$ is predominantly absent on the surface. The surface of the second sample has a structure of period of $50.8 \mathrm{~nm}$, or 19,700 line pairs $/ \mathrm{mm}$, in agreement with the $\Phi=7.8^{\circ}$ cut determined from dispersion measurements described later in Subsection 5.B.

The AFM images show terraced steps with quite irregular edges, which is not what is usually expected for a grating of any usable quality. In this case, however, the structure of importance is the underlying layered volume where the interference takes place. As can be seen in the height map, the edges of the steps mainly follow a constant height, and hence the irregularity of the step edges is because they simply contour the surface. Several large scratches can be seen, for instance. Any irregularity in the surface height merely acts as a refractive screen placed on top of the perfect structure [32]. The phase change caused by a dip in the surface height by $h$ is $h(n-1) / \lambda=h \delta / \lambda$ waves, where $n$ is the refractive index of the missing material. The phase change for $13.2 \mathrm{~nm}$ light, due to the entire thickness of Mo $\left(\delta_{\text {Mo }}=0.077\right)$ is less than 0.02 waves, for example. Such a surface imperfection may cause scattering of the reflected light, equivalent to a surface with roughness of only 0.01 waves, or $0.13 \mathrm{~nm}$. That is, unlike a conventional single-surface reflector, reflection from a multilayer structure depends primarily on the quality of the underlying structure and the top surface is only a small contributor to the reflection. Even so, the larger scale irregularities, such as scratches, that show up in the AFM maps could cause a noticeable reduction in reflectivity due to scattering. We can estimate the reduction in reflectivity due to this phase dis-
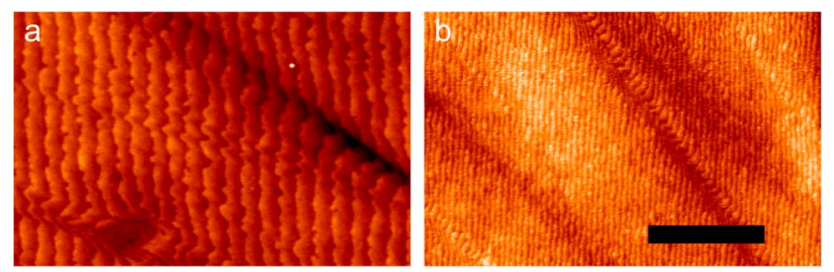

Fig. 4. (Color online) AFM height maps of the cut regions for a, the $\Phi=2.9^{\circ}$ cut and $\mathrm{b}$, the $\Phi=7.8^{\circ}$ cut. The scale bar is $1 \mu \mathrm{m}$, and the range of the color scale is $20 \mathrm{~nm}$ from lowest height (black) to highest (white). These correspond to blazed gratings of 7250 and 19,700 line pairs/mm, respectively. The grating efficiency is essentially insensitive to surface imperfections because x-ray diffraction is a volume effect. turbance by an exponential factor [33], as

$$
R=R_{B} \exp \left\{-\left(4 \pi \sigma_{s}(n-1) \cos \alpha / \lambda\right)^{2}\right\}
$$

where $\sigma_{s}$ is the RMS surface height variation. For the $7.8^{\circ}$ cut shown in Fig. $4 \mathrm{~b}$ we measure $\sigma_{s}=2.5 \mathrm{~nm}$ (from a $5 \mu \mathrm{m} \times$ $5 \mu \mathrm{m}$ area AFM scan), giving a reduction factor in reflectivity of 0.974 for $\lambda=13.2 \mathrm{~nm}$ and $n-1=0.077$. Clearly, this surface irregularity does not adversely effect the reflectivity of the cut multilayer.

\section{RESULTS AND DISCUSSION}

\section{A. Extreme Ultraviolet Reflectivity}

Measurements of the reflectivity of the cut $d=6.88 \mathrm{~nm}$ multilayers were performed at Bragg angles of $80.0^{\circ}$ (wavelength of $13.7 \mathrm{~nm}$ ) and $30.0^{\circ}$ (wavelength of $6.88 \mathrm{~nm}$ ) for comparison with theory. Some possible geometries are listed in Table 1 for measurements from the two cut angles of $\Phi=2.9^{\circ}$ and $7.8^{\circ}$, plus the symmetric reflection from the uncut portions $\left(\Phi=0^{\circ}\right)$. The measured reflectance as a function of wavelength, for a fixed incidence angle relative to the multilayer interfaces of $\theta=80^{\circ}$ and a detector with a $1.3^{\circ}$ acceptance, is shown in Fig. 3a, for the symmetric $(b=-1)$ and asymmetric $\left(b=-1.05, \Phi=7.8^{\circ}\right)$ reflections. The wavelength of the incident light was selected by a grating monochromator that is part of the beamline. The monochromator is designed to allow a continuous adjustment of the wavelength without changing the incidence angle on the sample.

The measured asymmetric reflectivity has a peak value of $51.4 \%$. For an asymmetry parameter $b=-1.05$, Eq. (17) predicts a reflectivity $R_{B}$ almost indistinguishable from the symmetric reflection on the plot of Fig. 2a. The reduction in peak reflectivity from the $53.7 \%$ of the symmetric reflection is presumably due to the difference in the surface structure caused by polishing. The reflectivity factor of 0.974 caused by the surface roughness of $2.5 \mathrm{~nm}$, as discussed in Section $\underline{4}$, gives a predicted reflectivity of $52.3 \%$ on the cut. That is, surface roughness partially explains the observed reduction in reflectivity. In addition to the roughness, however, the Mo layers that become exposed by the cut will completely oxidize and this incorporation of oxygen atoms will reduce the reflectivity. $\mathrm{A} \mathrm{MoO}_{3}$ oxide layer of thickness $4.3 \mathrm{~nm}$ and density $4.69 \mathrm{~g} \mathrm{~cm}^{-3}$, for example, would give rise to the observed reduction in reflectivity.

Measurements were carried out over a broad range of detector angles for a fixed incidence angle of $\alpha=-2.2^{\circ}$. At the

Table 1. Geometries of Some Reflections Possible for the $d=6.88 \mathrm{~nm}$ Multilayer with Cut Angles of $2.9^{\circ}$ and $7.8^{\circ}$

\begin{tabular}{ccccc}
\hline$\lambda$ & $\theta_{B}$ & $\Phi$ & $\phi$ & $b$ \\
\hline $13.7 \mathrm{~nm}$ & $80.0^{\circ}$ & $0^{\circ}$ & $-90^{\circ}$ & -1 \\
$13.7 \mathrm{~nm}$ & $80.0^{\circ}$ & $2.9^{\circ}$ & $-92.9^{\circ}$ & -0.982 \\
$13.7 \mathrm{~nm}$ & $80.0^{\circ}$ & $2.9^{\circ}$ & $-87.1^{\circ}$ & -1.018 \\
$13.7 \mathrm{~nm}$ & $80.0^{\circ}$ & $7.8^{\circ}$ & $-97.8^{\circ}$ & -0.953 \\
$13.7 \mathrm{~nm}$ & $80.0^{\circ}$ & $7.8^{\circ}$ & $-82.2^{\circ}$ & -1.050 \\
$6.88 \mathrm{~nm}$ & $30.0^{\circ}$ & $0^{\circ}$ & $-90^{\circ}$ & -1 \\
$6.88 \mathrm{~nm}$ & $30.0^{\circ}$ & $2.9^{\circ}$ & $-92.9^{\circ}$ & -0.834 \\
$6.88 \mathrm{~nm}$ & $30.0^{\circ}$ & $2.9^{\circ}$ & $-87.1^{\circ}$ & -1.192 \\
$6.88 \mathrm{~nm}$ & $30.0^{\circ}$ & $7.8^{\circ}$ & $-97.8^{\circ}$ & -0.616 \\
$6.88 \mathrm{~nm}$ & $30.0^{\circ}$ & $7.8^{\circ}$ & $-82.2^{\circ}$ & -1.622 \\
\hline
\end{tabular}


cut angle of $\Phi=7.8^{\circ}$, we have an equivalent grating period of $D=d / \cos (\Phi-\pi / 2)=50.7 \mathrm{~nm}$, where $d=6.88 \mathrm{~nm}$. If the grating was not perfectly blazed, then we would expect some diffraction at orders other than the first. The second order, for example, would occur at an angle given by $\sin \beta=$ $\sin \left(-2.2^{\circ}\right)-2 \cdot 13.2 \mathrm{~nm} / 50.7 \mathrm{~nm}$, or $2 \theta=143.8^{\circ}$. As clearly seen in Fig. 3b, no measurable diffraction above background could be detected.

\section{B. Dispersion}

From Eq. (16) it is clear that we may achieve dramatic demonstrations of dispersion for significant values of $|b+1|$. For our fabricated multilayer samples, this requires measurement at wavelengths much shorter than $13.7 \mathrm{~nm}$. At a wavelength of $6.9 \mathrm{~nm}$ the larger dispersion allows higher sensitivity measurements to be made for comparison with predictions. The multilayer materials are not optimized for the shorter wavelength, however, so the reflectivity is only $2 \%$, as measured. We measured the dispersion at a fixed incidence angle by scanning the detector angle $\beta$ and wavelength. The measured reflectivity as a function of $2 \theta=\alpha-\beta$ (with $\alpha$ constant) and $\lambda$ are shown in Fig. $\underline{5}$. The angular acceptance of the detector in the scan direction was $0.1^{\circ}$. The linear dependence of the angle of the peak reflectivity on the wavelength can clearly be seen.

For the measurement shown in Fig. 5a, we allowed a small portion of the incident beam to reflect from the uncut part of the multilayer. This portion is seen as the low-reflectivity peak that occurs at a constant $2 \theta$ for all values of $\lambda$. This has $b+1=$ 0 and no dispersion, as expected for a symmetric multilayer. The two beams reflecting from the cut and uncut parts of the multilayer intersect at $2 \theta=2 \theta_{B}$ as is necessary from the grating equation, Eq. (1), which can be rewritten as

$$
\sin \beta=\frac{-\lambda \cos \phi}{d}+\sin \alpha
$$

On the uncut portion of the multilayer $\cos \phi=0$ and $\operatorname{so} \alpha=\beta$. Because $\alpha$ is fixed, $\beta$ is fixed also, and this symmetric reflection defines the Bragg angle for this particular geometry as $2 \theta_{B}=2 \theta=\alpha-\beta$. For the cut multilayer, where $\cos \phi$ is nonzero, we see from Eq. (37) that $\sin \beta$ varies linearly with $\lambda$ and that when $\lambda=\lambda_{B}=2 d \sin \theta_{B}$ then $\sin \beta=-2 \sin \theta_{B} \cos \phi+$ $\sin \alpha$, for which $2 \theta=\alpha-\beta=2 \theta_{B}$, intersecting with the symmetric reflection. Note that the maximum reflectivity does not occur at the Bragg condition. This displacement of the peak from the Bragg wavelength is the refractive shift $\Delta \lambda_{s}$ [Eq. (23)], which can be measured here, because this intersection of the two beams is independent of the choice of $\phi$. The determination of $\lambda$ and $\theta_{B}$ at the intersection of the two reflected beams in Fig. 5a provides a way to measure $d$ without needing to know the materials properties of the multilayer. In particular we find that $\theta_{B}=30.39 \pm 0.03^{\circ}$ and $\lambda_{B}=6.96 \pm$ $0.02 \mathrm{~nm}$, giving $d=6.88 \pm 0.02 \mathrm{~nm}$. This is in agreement with the $6.876 \pm 0.005 \mathrm{~nm}$ period determined by a diffractometer measurement at $8.054 \mathrm{keV}$.

For each wavelength we determined the $2 \theta$ position of the peak reflectivity by a Gaussian fit. These positions are displayed on Fig. 5a as white diamonds (peaks for only every second wavelength value are displayed for clarity). The grating equation was then fit to the peak positions, varying $\phi$ and setting $d=6.88 \mathrm{~nm}$. The best fit was found for $\phi=-81.2^{\circ}$ or a
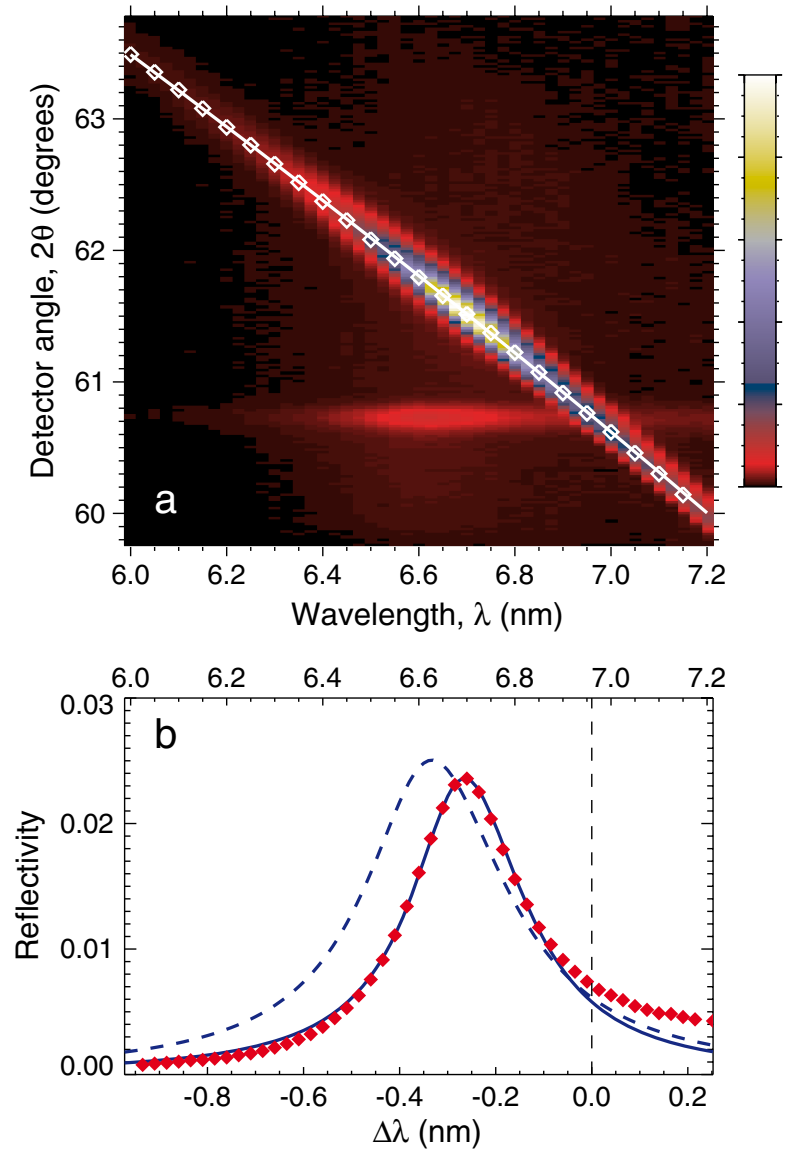

Fig. 5. (Color online) a, Reflectivity of the $\Phi=7.8^{\circ}$ cut mirror at a Bragg angle of $30.39^{\circ}, b=-1.49$, as a function of detector angle $2 \theta$ and wavelength $\lambda$. The incidence angle was $\alpha=-53.0^{\circ}$. Positions of the peak reflectivity at each wavelength are displayed with diamonds, and the line is a fit of the grating equation, Eq. (1), to these peaks. $\mathrm{b}$, Reflectivity, integrated over $2 \theta$, from a dataset similar to that shown in panel a is displayed with diamonds The solid line is a calculation from Eq. (17), scaled by a factor 0.53 , and the dashed line is a calculation for the symmetric reflection using the IMD program and applying the same scaling factor.

cut angle of $\Phi=7.8^{\circ}$. This is in agreement with the AFM measurement, as mentioned in Subsection 4.B. We carried out the same procedure on the $\Phi=2.9^{\circ}$ cut multilayer and found a similar agreement between the inferred cut angle and the period determined by AFM.

In Fig. $5 \mathrm{~b}$ we show the reflectivity of the cut multilayer as a function of wavelength, determined by integrating over the $2 \theta$ scans for each wavelength, and normalizing by the integrated scan over the incident beam. This was performed for a $2 \theta-\lambda$ dataset where the full beam was reflected by the cut portion of the multilayer. The maximum reflectivity is $2.36 \%$, occurring at $\Delta \lambda=-0.26 \pm 0.01 \mathrm{~nm}$. This compares with $\Delta \lambda_{s}=-0.251 \mathrm{~nm}$, calculated from Eq. (23) with the optical constants of the multilayer materials given in Section 3 . Using the multilayer composition of repetitions of the four layers plus the termination with $4.8 \mathrm{~nm}$ thick $\mathrm{SiO}_{2}$, determined by fitting the $13.2 \mathrm{~nm}$ reflectivity (Subsection 4.A), we calculate from the IMD program [20] a peak reflectivity of the symmetric reflection of $2.5 \%$. (Without the oxide, the reflectivity is calculated to be 4.7\%.) The calculation of the reflectivity curve for the cut multilayer with $b=-1.49$, from Eq. (17) using the same multilayer parameters and replacing Eq. (34) with a four-layer 
model with layer interdiffusion, predicts a maximum reflectivity of $4.4 \%$. This reflectivity curve is shown in Fig. 5a with a solid line, after scaling the reflectivity by a factor of 0.53 to account for the surface oxide. At this wavelength the reflectivity is a strong function of the oxide layer thickness and the interdiffusion length, and we can also obtain a good fit to the measured data by varying the oxide layer thickness by $1 \mathrm{~nm}$ instead of scaling. Nevertheless, the oxide thickness as predicted from the $13.2 \mathrm{~nm}$ measurements does fit the results at $6.9 \mathrm{~nm}$ wavelength. The reflectivity curve of the symmetric reflection, calculated from Eq. (17) and with the same reflectivity factor of 0.53 is shown in Fig. $5 \mathrm{~b}$ with a dashed line. We note that the shape of the calculated reflectivity curve of the asymmetric reflection agrees well with the measurement, except at $\Delta \lambda>0$, where the measured reflectivity is higher. The calculated refractive shift $\Delta \lambda_{s}$ and the Darwin width $\Delta \lambda_{w}$ are in agreement with the measurement.

The angular width of the diffracted beam depends on the spectral resolution of the cut multilayer grating. The measurement of this width at the wavelength of maximum reflectivity is taken from a vertical line-out of the plot in Fig. 5 at a wavelength of $6.7 \mathrm{~nm}(\Delta \lambda=-0.26 \mathrm{~nm})$. The full-width at half-maximum (FWHM) of the dispersed beam is $3.7 \mathrm{mrad}$ and the FWHM of the specularly reflected beam is $2.8 \mathrm{mrad}$. The angular width of the specular beam gives an estimate of the angular extent of the detector slit, and deconvolving this from the measurement of the dispersed beam gives a FWHM of $0.9 \mathrm{mrad}$. This corresponds to a spectral resolving power of $\lambda / \Delta \lambda=\tan \theta / \Delta \theta \approx 700$ [see Eq. (16)]. The bandwidth of the incident light, set by the grating and monochromator slits of the diffractometer [27] was $\Delta \lambda / \lambda=1 / 1000$. Assuming this and the contribution to the cut multilayer add in quadrature then we infer that the intrinsic resolving power of the cut multilayer is also $\lambda / \Delta \lambda=1000$. This is lower than the upper bound of a resolving power of 2000 set by the total number of periods in the grating. The lower resolving power could be attributed to an accumulated error in position and period of the layers in the grating structure, or other unaccounted instrumentation errors in our measurement.

\section{APPLICATIONS OF SLICED MULTILAYERS}

\section{A. Monochromator or Spectrometer}

The symmetric Bragg reflection from a multilayer coating $(b=-1)$ can of course be used as a monochromator (a bandpass filter). However, in that case the bandwidth depends on the Darwin width $\Delta \lambda_{w}$ which is essentially the inverse of the number of layers that contribute to the reflection. The bandwidth can be easily increased by reducing the number of layers in the multilayer stack to less than the extinction depth $\Lambda_{0} / d$, but decreasing the monochromator bandwidth requires thick multilayers with materials of low absorption so that the $\mathrm{x}$-ray wave field interacts with the entire stack. That is, the smallest bandwidth that can be achieved with an uncut multilayer is dependent on materials properties. It is clear that a cut multilayer gives much greater freedom in designing a monochromator, because this structure is essentially a dispersing grating. A cut multilayer also can be used as a spectrometer, dispersing a portion of the incident spectrum within the multilayer's Darwin width. The smallest monochromator bandwidth or spectrometer resolution is limited by the number of illuminated grating periods. Because the grating period $D$ can easily be much smaller than gratings made by other means, more periods could be illuminated than for a conventional grating in a similar geometry. (Or equivalently, the dispersion of the cut multilayer can be much higher than a conventional grating.) Generally, a cut multilayer may be advantageous for monochromator bandwidths between $10^{-2}$ to $10^{-4}$, especially for wavelengths longer than several angstrom where crystals might not be appropriate.

A practical limitation to the use of a cut multilayer is that caused by their limited thickness. The 2020-period multilayer reported here is one of the thickest made, and yet when cut at an angle $\Phi=7.8^{\circ}$ intersects a beam height of only $110 \mu \mathrm{m}$. The effective height of the cut can be increased by cutting or etching a sawtooth pattern into the multilayer stack to produce a so-called Bragg-Fresnel multilayer optic [34,35]. These types of structures have achieved bandwidths of $\overline{10}-\overline{36}$. For $b=1$, Laue reflection, a rectangular pattern could be etched into the stack to leave a series of multilayer walls on a substrate. This structuring for Bragg or Laue reflection effectively increases the number of interacting periods, which would decrease the bandwidth if the cuts were all of the same angle. The layers of the structure would all be in phase from one cut to another, but small changes in the cut angles would lead to changes in $b$ and the dispersion in these cuts. From Eq. (16) the variation in $b$ should be less than the desired bandwidth: $\Delta b<(\Delta \lambda / \lambda)(1+b)$.

\section{B. Phase Corrector Plates}

The option exists of making a profile other than a straight cut in the multilayer. Cutting a curved profile results in a grating profile on the curved surface with a variable line spacing. The line spacing is related to the surface shape (the lines are contours of the shape). A fruitful way to examine multilayers (e.g., deposited on a flat substrate) with large-scale curved shapes cut out of them is to note that the wave field interacts with the multilayer structure and the phase of the wave field is "pinned" to the layers. Thus, where the height of the terminating surface of the multilayer changes there will be a different path length of the reflecting beam as it either travels through the multilayer material or vacuum (the region that has been cut away from the multilayer). For example, in a step of height $h$ cut into a multilayer that exceeds this height, the phase of the Bragg-reflected beam is pinned by the same common multilayer on both sides of the step that exists below the cut. The only difference between the reflected beams on either side of the step is that the incident and reflected waves travel through a height $h$ of vacuum (of refractive index 1) in the milled region, or a height $h$ of multilayer (with effective refractive index $n_{m} \approx 1+\chi_{m} / 2$ ) in the unmilled region, before reaching the depth where the multilayer is identical. The difference in the phase of the beam reflecting from the etched region to the beam reflecting from the unetched region is $4 \pi\left(1-n_{m}\right) h / \lambda$. For x rays, which have $\chi_{m}<0$ and $1-n_{m}>0$, a spherical depression milled out of a multilayer will act as a positive refractive lens. However, this lens will be weaker in power (by a factor of $1-n_{m}$ ) as compared to a substrate of the same shape that has been coated with a multilayer. The advantage for the manufacture of optics is that the tolerance for surface imperfections is increased by this same factor. 
From Eq. (21) the phase difference between beams reflected from a step height equal to the extinction depth $\Lambda_{0}$ will be $2 \pi\left(\chi_{m} \chi_{\bar{m}}\right)^{1 / 2} \Lambda_{0} / \lambda=2 \pi\left|\gamma_{0}\right|$. Thus, it is not possible to generate phase differences much larger than $2 \pi$. A general profile can be made by cutting the shape, modulo $2 \pi$. This is the basis of the Bragg-Fresnel lens. It is easier, however, to fabricate a binary structure, leading to a zone-plate lens rather than a Fresnel lens.

Another possibility to achieve a variable line space is to vary the $d$ spacing of the multilayer. Point-to-point focusing can be achieved this way by ensuring the multilayers form a volume hologram caused by the interference of spherical waves emanating from those points. These volume zone plates are discussed in Subsection 6.D.

\section{Pulse Compressor}

\section{Chirped Pulses}

The wavelength dispersion of sliced multilayers can be used to construct optical systems that give a variation of path length with wavelength but which preserve the collimation of a beam. These can be used to induce a linear frequency chirp on a short pulse or to compress a frequency chirped pulse. X-ray free-electron lasers can in principle be configured to produce chirped pulses, which could then be optically compressed [37]. X-ray pulse compressors based on a pair of gratings [37] or a strained crystal [38] have been proposed. A sliced multilayer pulse compressor has the advantage of high efficiency. It may be easier to fabricate than a strained crystal and be more practical than a pair of lower dispersion gratings (which will require a longer length between the elements and produce a larger shear of the beam for a given compression).

A pulse with a linear chirp can be described as pulse with a wavelength that varies with position $x$ along the pulse (or time $t=x / c$ where $c$ is the speed of light) as

$$
\lambda(x)=\lambda_{0}\left(1-\gamma_{c} x / L_{p}\right)
$$

where $L_{p}$ is the length of the pulse (the pulse duration is $L_{p} / c$ ) and $\gamma_{c}=\Delta \lambda / \lambda$ is the relative bandwidth of the chirp. We define $x=0$ as the leading edge of the pulse. The chirp may be described as positive, where the leading edge of the pulse has a longer wavelength $\left[\lambda(0)=\lambda_{0}\right]$ than the trailing edge $\left[\lambda\left(L_{p}\right)=\lambda_{0}\left(1-\gamma_{c}\right)\right]$. The pulse compressor must produce a path that compensates for this variation, by directing the longer wavelength light along a longer path, so that the tail catches up with the head:

$$
x_{c}(\lambda)=x_{0}+L_{p}\left(\lambda-\lambda_{0}\right) / \lambda\left(1 / \gamma_{c}\right) \text {, }
$$

or

$$
\Delta x_{c}(\Delta \lambda)=L_{p}\left(\frac{\Delta \lambda}{\lambda}\right) / \gamma_{c}
$$

In the case of a negative chirp, $\gamma_{c}<0$, the compressor must create a longer path for the shorter wavelength light to compress the pulse. As an example of chirp parameters, the Linac Coherent Light Source (LCLS) [39] produces high-fluence pulses between 70 and $400 \mathrm{fs}$ duration $\left(L_{p}\right.$ between 20 and $120 \mu \mathrm{m})$ and could be configured to produce a chirp of about $\gamma_{c}=0.5 \%$.

\section{Compressor Geometry}

A pulse compressor can be made with two sliced multilayers, either in the Bragg or Laue geometry (Figs. 6 and 7). Rays of different wavelength incident on the first element will reflect according to the reflectivity curve and be dispersed according to the grating equation as, for example, shown in Fig. $\underline{5}$. These rays are recollimated by the second element. That is, we have $\alpha_{2}+\beta_{1}=-\pi$ and $\beta_{2}+\alpha_{1}=-\pi$, which implies that $b_{1}=1 / b_{2}$, the multilayers must be of the same period $d$ and cut angle $\phi$ and that the multilayer surfaces must be parallel. Here the subscripts refer to the first and second elements. In the following, when no numerical subscripts are used, we refer to the geometry of the first element. Rays impinging on the first element at the same position will be spread by an amount $S$, which ideally should be much less than the width of the incident beam. To correct for this shear of the beam a symmetric layout of four elements may be used, by mirroring the setup about a plane perpendicular to the output ray direction (Fig. 8). This, however, will result in reduced efficiency and increased complexity.

Consider the Bragg compressor shown in Fig. 6, with two sliced multilayers separated by a distance $H$ in the direction of the surface normals. The case for $b<-1$ is shown. The distance of the reflected ray, from the point of reflection from the first multilayer to the point of intersection with the second multilayer, projected on to the surface of the multilayers is $L=H \tan \beta$, and thus

$$
\frac{\partial L}{\partial \beta}=\frac{H}{\cos ^{2} \beta} .
$$

Note that the sense of the angles is as defined by the Bragg angle being positive, $\theta_{B}=\left(\mathbf{s}, \mathbf{s}_{0}\right)$ as described in Section 2 . That is, a positive increase in $\beta$ increases $L$. The difference in path length between the two rays reflected from the second multilayer will be

$$
\Delta x_{c}=\Delta L(\sin \alpha-\sin \beta)
$$

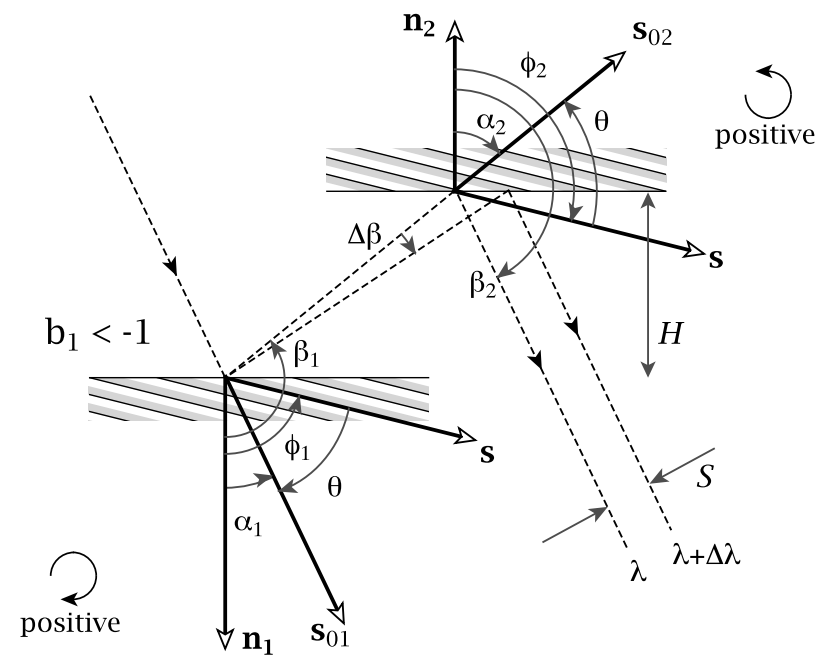

Fig. 6. Geometry of a pulse compressor in Bragg geometry for the case that $b<-1$. Ray paths are indicated by dashed lines. The circular arrows labeled "positive" show the direction of positive angles for the two reflections. 


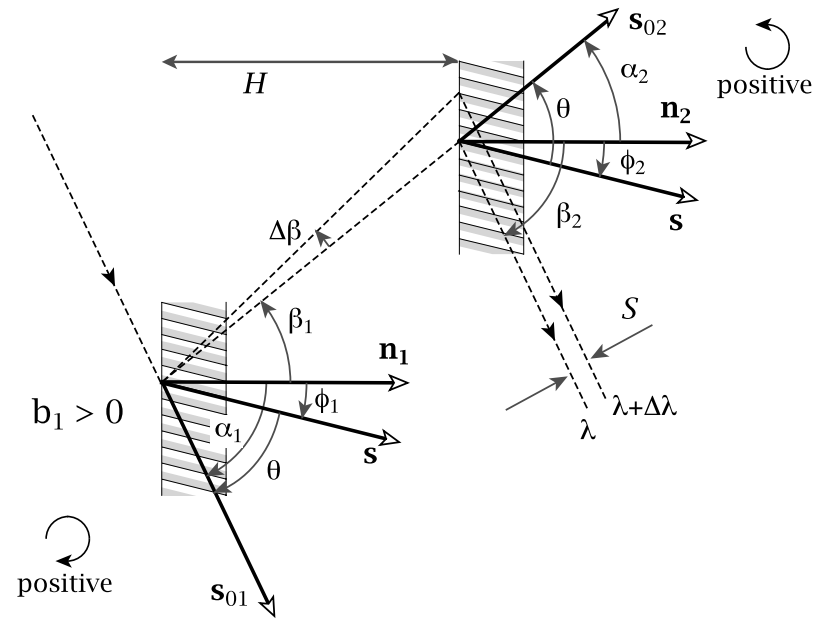

Fig. 7. Geometry of a pulse compressor in Laue geometry, $b>0$. Ray paths are indicated by dashed lines. The circular arrows labeled "positive" show the direction of positive angles for the two reflections.

Combining Eqs. (2), (16), and (41), Eq. (42) can be written as

$$
\Delta x_{c}=-2 \frac{\Delta \lambda}{\lambda} \frac{H}{\cos ^{2} \beta}(1+b) \tan \theta_{B} \cos \phi \sin \theta_{B} .
$$

This same equation is valid for the Bragg geometry with $-1<b<0$, even though the dispersion is reversed. The equation also applies, except for a sign change, for the Laue case of Fig. 7, for a distance $H$ between the parallel multilayers. Noting that $1+b=2 \cos \theta \cos \phi / \cos \beta$, we find that the general expression for both the Bragg and Laue cases can be expressed as

$$
\Delta x_{c}=\left(\mathbf{n}_{1} \cdot \mathbf{n}_{2}\right) \frac{\Delta \lambda}{\lambda} \frac{H}{\cos \beta}(1+b)^{2} \tan \theta_{B} .
$$

As is seen from Eq. (44) the path difference $\Delta x_{c}$ varies linearly with the relative wavelength change $\Delta \lambda / \lambda$, as required by Eq. (40), and on the distance along the ray path between the elements, $|H / \cos \beta|$. Additionally, the path difference depends on the properties of the sliced multilayer, characterized by the asymmetry parameter $b$ and Bragg angle $\theta_{B}$. By comparing with Eq. (16) we see that $x_{c}$ is proportional to the square of the dispersion $\partial \beta / \partial \lambda$. The largest chirp for a given wavelength $\lambda$ is therefore achieved with the largest Bragg angle (or smallest multilayer period) and for the largest value of $|1+b|$. For compressors in either the Bragg or Laue geometries, $|1+b|$ is maximized for an incident beam normal to the surface which asymmetrically reflects at an angle grazing the surface. That is, the cut angle $\Phi$ would be near $45^{\circ}$. However, we note from Eq. (24) that the bandwidth of the multilayer decreases with increasing $|b|$ as $|b|^{-1 / 2}$. The asymmetry pa-

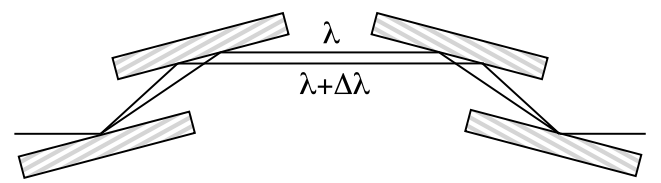

Fig. 8. Four sliced multilayer elements arranged to compress pulses and correct for the beam shear. The geometry shown here has $-1<b<0$ rameter must be chosen to pass the relative wavelength range of the chirped pulse, $\gamma_{c}$, which will then dictate the length $H$ of the compressor for a given pulse length.

Equation (44) shows that sliced multilayer pulse compressors only compress pulses with a positive chirp. For Bragg geometry, defined when the reflected ray exits on the opposite side of the surface as the inward facing normal $\mathbf{n}, \cos \beta=\mathbf{n}$. $\mathbf{s}_{m}$ is always negative. Because the Bragg compressor has $\mathbf{n}_{1} \cdot \mathbf{n}_{2}=-1$, we have $\Delta x_{c} / \Delta \lambda>0$ and the compressor provides a longer path length for longer wavelengths, compressing a positively chirped pulse. For Laue geometry, $\cos \beta$ is positive by definition, $\mathbf{n}_{1} \cdot \mathbf{n}_{2}=1$, and so again $\Delta x_{c} / \Delta \lambda>0$. The relationship between path length and wavelength can be easily confirmed from ray diagrams such as shown in Fig. 6 .

The displacement of the beam after the two reflections of the compressor is given by

$$
\Delta y=-(H / \cos \beta) \sin 2 \theta_{B} .
$$

The shear $S$ induced on the beam is given by

$$
S=\Delta L \cos \beta_{2}=-\Delta L \cos \alpha,=\Delta x_{c} \frac{b}{1+b} \cot \theta_{B}
$$

The magnitude of the shear therefore increases with increasing $x_{c}$, but is minimized as $b \rightarrow 0$, which is the case of grazing incidence and near-normal reflection from the first element. The shear is negative for $-1<b<0$ and positive otherwise. A positive shear is defined as an increase in the displacement of the beam for longer wavelengths, as shown in Fig. 6 .

Depending on the application, the shear $S$ should be reduced to be much smaller than the width of the beam. In some instances, however, the widening of the beam by $S$ may be beneficial. For example, if the collimated beam was focused by a Kirkpatrick-Baez (K-B) mirror pair, the stretching of the beam in one direction by the compressor could compensate for the larger focal length of one of the mirror pairs relative to the other. If the stretching factor $(S+W) / W$ (where $W$ is the width of the beam incident on the pulse compressor) is equal to the ratio of focal lengths $f_{V} / f_{H}$, for instance, then the numerical aperture of the focused beam would be the same in the horizontal and vertical directions. Here it is assumed that the shear occurs in the vertical. If the K-B system was diffraction limited, then equalizing the vertical and horizontal numerical apertures produces a focal spot of the same vertical and horizontal dimensions.

\section{Compressor Efficiency}

For a perfect linear dependence of the wavelength on position along the pulse, the degree of compression depends on the collimation of the incident beam. An error in the incidence angle of $\Delta \alpha$ corresponds to a relative change in wavelength of $\Delta \lambda / \lambda=-\Delta \alpha \cot \theta_{B}$, corresponding to an equivalent error in the chirp correction. As an example, for a divergence of the incident beam of $1 \mu \mathrm{rad}$ for a wavelength of $0.15 \mathrm{~nm}$ and Bragg angle of $2^{\circ}$, the wavelength chirp error will be less than $0.003 \%$. This implies that a pulse with a perfect chirp of $0.5 \%$ bandwidth, for example, could be compressed by a factor of 160 .

For free-electron laser (FEL) sources, the degree of compression of the pulse depends upon the purity of the chirp of the incident pulse. Because of the limited longitudinal coherence of pulses produced by self amplification of spontaneous 
Table 2. Parameters of Pulse Compressors ${ }^{a}$

\begin{tabular}{lccccccccc}
\hline$\lambda(\mathrm{nm})$ & $d(\mathrm{~nm})$ & $\theta_{B}$ & $\Phi$ & $b$ & $H(\mathrm{~mm})$ & $\Delta y(\mathrm{~mm})$ & $|S|(\mathrm{mm})$ & $\Delta \lambda_{w} / \lambda$ & $R^{2}$ \\
\hline 0.15 & 2.00 & $2.1^{\circ}$ & $-1.0^{\circ}$ & -2.74 & 28 & 105 & 1.25 & $0.6 \%$ & 0.65 \\
0.15 & 2.00 & $2.1^{\circ}$ & $+1.0^{\circ}$ & -0.37 & 577 & 786 & 0.45 & $1.6 \%$ \\
0.15 & 2.00 & $2.1^{\circ}$ & $+1.5^{\circ}$ & -0.18 & 398 & 469 & 0.63 & 0.17 & $2.3 \%$ \\
$0.15^{b}$ & 2.00 & $2.1^{\circ}$ & $-90.0^{\circ}$ & +1.00 & 1060 & 79 & 0.46 & 0.40 & $1.0 \%$ \\
6.90 & 6.90 & $30^{\circ}$ & $-7.8^{\circ}$ & -1.62 & 17 & 40 & 0.47 & 0.13 & $3.0 \%$ \\
6.90 & 6.90 & $30^{\circ}$ & $-15.0^{\circ}$ & -2.73 & 1.5 & 5.2 & 0.26 & 0.08 & $2.0 \%$ \\
\hline
\end{tabular}

${ }^{a} \lambda$ is the wavelength, multilayer period $d$, and asymmetric-cut angle $\Phi=\pi / 2-\phi$, giving a Bragg angle $\theta_{B}$ and corresponding asymmetry parameter $b . H$ is the multilayer displacement required to compress a $100 \mathrm{fs}$ pulse with a chirp of $0.5 \%$, and $\Delta y$ and $|S|$ the resulting beam displacement and shear, respectively. The Darwin width $\Delta \lambda_{w}$ gives the maximum chirp that the compressor can efficiently compress. The materials for the $2 \mathrm{~nm}$ period are W/SiC with $\Gamma=0.22$ and Ru/ $\mathrm{B}_{4} \mathrm{C}$ with $\Gamma=0.31$ for the $6.9 \mathrm{~nm}$ period multilayers.

${ }^{b}$ This row is for the Laue geometry of Fig. 7 .

emission in an FEL, unchirped pulses have a limited bandwidth. This is referred to as the homogeneous bandwidth and is related to the FEL parameter [40]. For the LCLS at $\lambda=0.15 \mathrm{~nm}$ this homogeneous bandwidth is given by $\gamma_{n}=\Delta \lambda / \lambda=0.05 \%$. Any subpulse of the chirped beam will have this same homogeneous bandwidth. The maximum compression ratio for a pulse with a chirp $\gamma_{c}$ will therefore be $\gamma_{c} / \gamma_{n}$. A 10:1 compression ratio, required to reduce the pulse duration at LCLS from 100 fs to 10 fs, for example, thus requires a bandwidth acceptance of $\Delta \lambda_{w} / \lambda$ of at least $0.5 \%$.

As can be seen from Eq. (24), for a given multilayer material and period, the sliced multilayer bandwidth $\Delta \lambda_{w} / \lambda$ increases for increasing wavelength (due to the $\lambda^{2}$ dependence of susceptibility away from absorption edges) and for decreasing magnitude of the asymmetry parameter, $|b|$. A small value of $|b|$ corresponds to the expanding geometry where the width of the reflected beam is greater than that of the incident beam (grazing incidence). However, as seen in Fig. 2, decreasing $|b|$ may lead to a lower reflectivity, depending on the absorption of the multilayer materials.

The Laue geometry has the advantage of producing a larger chirp correction for a given instrument length $H$, due to the variation of the dispersion on $(1+b)^{2}$. However, for highly asymmetric condensing reflections $(|b| \gg 1)$ the Bragg and Laue geometries give approximately equivalent chirp corrections. Depending on the materials, higher reflectivity might also be achievable with Laue rather than Bragg reflection. Some parameters of pulse compressors are listed in Table 2, calculated for two wavelength ranges and for various multilayer materials. The Bragg or Laue reflectivity, $R_{B}$ or $R_{L}$, of a sliced multilayer is computed from Eqs. (17) or (25) as a function of the parameters $b, \theta_{B}$, and $\Delta \theta$ (or $\Delta \lambda$ ). The particular geometry that these correspond to can be computed from Eqs. (13-15). Given a particular geometry of incidence angle $\alpha$, asymmetry angle $\phi$, and wavelength $\lambda$, the reflectivity can be computed using $\theta_{B}=\alpha-\phi, \lambda_{B}=2 d \sin \theta_{B}$, and $\Delta \lambda=\lambda-\lambda_{B}$. By reciprocity, the reflectivity of the second sliced multilayer will be the same as the first. Thus the reflectivity of the pair is given by $R^{2}$, and the bandwidth is smaller than the single-reflection bandwidth $\Delta \lambda_{w}$, depending on the shape of the reflectivity curve.

In Table 2 , the bandwidth $\Delta \lambda_{w} / \lambda$ of a single reflection is listed, and the length $H$ required to compress a pulse of length $L_{p}=29 \mu \mathrm{m}(100 \mathrm{fs})$ and a chirp $\gamma_{c}=0.5 \%$. The shear $S$ is also listed, which can be compared to a beam size of approximately $1 \mathrm{~mm}$ for a beam of wavelength $0.15 \mathrm{~nm}$ that has propagated $400 \mathrm{~m}$ from the LCLS source. The table also lists parameters of some soft-x-ray compressors assuming the same chirp conditions. The $0.5 \%$ chirp can be accepted by the bandwidth of all cases listed in the table.

\section{Volume Zone Plate}

Zone plates are diffractive optical elements that provide constructive interference for ray paths that converge onto an image point and hence act as lenses. A Fresnel zone plate is a phase or amplitude screen with zones that alternately $\pi$-phase shift or block paths that differ in path length from the previous zone by $\lambda / 2$. The zone boundaries are given by $r_{n}^{2} \approx n f \lambda$, where $f$ is the focal length. To focus a collimated beam, a zone at radius $r$ must deflect a ray by an angle $2 \theta \approx r / f$ such that it intersects the axis a distance $f$ from the element. That is, a structure of period $d=\lambda /(2 \sin \theta)$ is required at that radius, or $d(r) \approx \lambda f / r$.

Fresnel zone plates are used very successfully in soft-x-ray microscopy $[\underline{41}, \underline{42}]$. At harder wavelengths, thin Fresnel zone plates are not efficient and a volume diffractive element is required. This is clear from a plot of the Laue reflectivity $R_{L}$ of a $\mathrm{W} / \mathrm{SiC}$ multilayer, as a function of thickness $t$ for a wavelength of $0.15 \mathrm{~nm}$ and $d$ spacing of $2 \mathrm{~nm}$, shown in Fig. 9 . In this case the reflectivity is maximum for a thickness of $t=4.95 \mu \mathrm{m}$, approximately equal to half the Pendellösung distance $\Lambda_{0}=$ $10.7 \mu \mathrm{m}$, Eq. (21). This optimum thickness is almost 2500 times the period of the structure. For this particular example, the Bragg angle is equal to $\theta_{B}=2.1^{\circ}$. It is clear that a ray entering the zone plate at this point can interact with as many as $t \tan 2 \theta_{B} / d=186$ layers. Such focusing elements can be fabricated by multilayer deposition followed by slicing, and designs can be made in the reflective (Bragg) or transmissive (Laue) geometry. Volume elements based on sliced variableperiod multilayered structures have been fabricated and

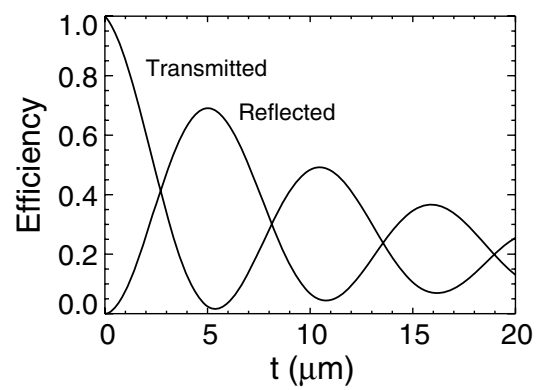

Fig. 9. The calculated efficiency of the reflected and transmitted beams from a cut W/SiC multilayer slab of thickness $t$ in the symmetric Laue geometry (e.g., the local zone-plate efficiency), with $b=+1$ for a wavelength of $\lambda=0.5 \mathrm{~nm}$. The multilayer period is $d=2 \mathrm{~nm}$, and the reflectivity is optimized at $\Gamma=0.22$. 
Table 3. Reflectivities of Various Sliced Multilayers in the Symmetric Laue Geometry $(b=1)$

\begin{tabular}{|c|c|c|c|c|c|c|}
\hline Material Combination & $d(\mathrm{~nm})$ & $\Gamma^{a}$ & $\lambda(\mathrm{nm})$ & $t_{L}(\mu \mathrm{m})$ & $\Lambda_{0}(\mu \mathrm{m})$ & $R_{L}$ \\
\hline W/SiC & 2.0 & 0.22 & 0.15 & 4.95 & 10.6 & 0.69 \\
\hline W/SiC & 10.0 & 0.22 & 0.15 & 4.96 & 10.6 & 0.69 \\
\hline W/SiC & 2.0 & 0.13 & 0.06 & 18.9 & 39.6 & 0.76 \\
\hline W/SiC & 2.0 & 0.50 & 0.06 & 7.25 & 15.7 & 0.67 \\
\hline $\mathrm{Mo} / \mathrm{B}_{4} \mathrm{C}$ & 2.0 & 0.10 & 0.15 & 17.8 & 37.2 & 0.75 \\
\hline $\mathrm{C} / \mathrm{B}_{4} \mathrm{C}$ & 2.0 & 0.34 & 0.15 & 211 & 430 & 0.92 \\
\hline $\mathrm{Al}_{2} \mathrm{O}_{3} / \mathrm{B}_{4} \mathrm{C}$ & 2.0 & 0.50 & 0.15 & 24.2 & 50.0 & 0.87 \\
\hline
\end{tabular}

${ }^{a}$ The ratio $\Gamma$ of material thicknesses was optimized to give the highest Laue reflectivity $R_{L}$, except in the cases of $\Gamma=0.5$, which minimizes the thickness $t$.

tested. Called multilayer Laue lenses, they have been used to produce the smallest one-dimensional focal spots of $\mathrm{x}$ rays [43-45]. Two-dimensional focusing can be achieved with two orthogonal Laue lenses, or in principle from a circular "jelly-roll" type lens [46] with concentric conical layers.

It is well appreciated that high efficiency of thick zone plates requires that the Bragg condition be satisfied [14,47], requiring a tilt of the layers, which depends on the layer radius. As with all volume holograms [48], this tilt is specific for one source and image point pair, and efficiency diminishes when used in other configurations or over an extended field. For the case of focusing a collimated beam, the tilt of the layers is given by $\theta \approx r /(2 f)$. (Focusing from a source point a distance $2 f$ from the zone plate to $2 f$ away does not require any tilt of the layers.) The advantage of the volume effect of the reflection, just as with reflection from any other sliced multilayer system, is that the efficiency of the reflection can reach $100 \%$, which means that the unreflected transmitted intensity (the zero-order diffraction of the zone plate) can approach zero. Unlike the thin zone plate there are no negative or higher order focii.

The dynamical diffraction theory applied in this paper is valid only for multilayers of a single period. Several analyses have been made for volume zone plates, based on coupled-wave theories $[14,15,47]$. Distorted arrays, such as the $1 / r$ dependence of the period, have been modeled by the Tagaki-Taupin theory [49]. Here we simply apply the single-period dynamical diffraction theory locally to a small patch of zone plate where we assume the period does not change. In this way we can estimate the zone-plate efficiency as a function of reflection angle and determine the optimum thickness profile of the plate.

Table 3 lists the Laue reflectivities for various geometries, multilayer materials, and wavelengths, for lenses of optimal thickness for that particular $\theta_{B}$. The efficiency of the Laue reflection increases with diminishing absorption, and so higher efficiencies are generally obtained with shorter wavelengths. Thus, volume zone plates complement thin Fresnel zone plates which are efficient at longer wavelengths. As expected, the optimum thickness, $t_{L}$, increases for decreasing wavelength.

We find from calculations that the optimum thickness is remarkably insensitive to $d$ spacing, for all spacings greater than about $0.1 \mathrm{~nm}$. The optimum thickness is dependent on the ratio of materials, $\Gamma$, and the minimum of $\Lambda_{0}$ is found at $\Gamma=0.5$ (for multilayers consisting of two materials). The optimum thickness does depend on the asymmetry parameter $b$, however. In a volume zone plate that focuses a collimated beam, $\alpha=0$ and the Bragg condition is satisfied by tilting zones according to $\phi=-\theta_{B}$. The asymmetry parameter is then $b=1 / \cos \beta=1 / \cos 2 \theta_{B}$. Given that $\tan 2 \theta_{B}=r / f$ we have

$$
b=\sqrt{1+r^{2} / f^{2}} .
$$

The asymmetry parameter will only vary appreciably for high numerical apertures above 0.5 . Therefore, for practical applications, the optimum profile of a volume zone plate is of constant thickness.

\section{CONCLUSIONS}

Sliced multilayer optics are equivalent to perfect multilayercoated blazed gratings. The line spacing of the grating is determined by the multilayer period formed by deposition of materials. Because periods down to several angstrom can be fabricated, grating linewidths approaching this value can be made. This allows for dispersions of $\mathrm{x}$ rays that are much higher than achievable gratings made by ruling or lithography. We have provided an analysis of asymmetric-cut multilayers by applying a dynamical diffraction theory of periodic structures. This allowed us to describe the properties of blazed gratings in terms of the asymmetry parameter $b$, providing a unified framework to characterize diffracting structures in both the reflecting Bragg and transmitting Laue geometries. The asymmetry parameter and the Bragg angle completely specify the geometry of a transmission or reflection grating but we have found that it is often confusing to convert this into a description of experimental parameters. We present solutions to determine the blaze angle in terms of asymmetry parameter in all geometries of flat transmission and reflection gratings.

We made grating structures by an asymmetric cut in a thick $\mathrm{x}$-ray multilayer structure. These gratings were characterized with soft $\mathrm{x}$ rays at the Advanced Light Source reflectometer. We find that the performance of the grating does not depend critically on the surface structure exposed by cutting and polishing the multilayer because the dispersion and reflection of the light is a volume effect. Creating a high-quality optic does of course require that the entire multilayer stack is grown without an interruption in its period and is thick enough to expose the cut surface. Growing such thick structures requires conditions that produce low stress and low roughness stacks.

We studied the efficiency of Mo/Si multilayer with 2020 bilayers with a period thickness of $6.88 \mathrm{~nm}$ and cut under different angles. Fabrication of such a thick multilayer structure took about $48 \mathrm{~h}$, during which time some interdiffusion took place, due to a raised substrate temperature. In addition to accumulated roughness this interdiffusion can explain the measured normal-incidence reflectivity of $53.7 \%$. The reflectivity from the asymmetric cut is somewhat lower (51.4\%), most likely due to oxidation of the exposed Mo layers as well as surface imperfections due to cutting and polishing. 
Nevertheless, the performance of the grating structures agrees well with the dynamical diffraction theory predictions. For example, measurements over a broad range of angles only revealed diffraction in the first diffraction order, indicative of the inherent perfect blaze achieved by this method. No higher diffraction order peaks could be detected. Our experiments carried out at EUV wavelengths give credence to our simulated results at shorter wavelengths. At shorter wavelengths, absorption is lower and the dynamical diffraction theory is even more accurate.

The thick multilayer cut at an angle of $\Phi=7.8^{\circ}$ is equivalent to a blazed grating with $\approx 20,000$ line pairs $/ \mathrm{mm}$ with greater than $90 \%$ relative efficiency ( $>51 \%$ absolute efficiency). This is higher efficiency than can be achieved by lithographic methods [6,7]. A single asymmetric cut only gives a limited grating area, compared to lithographic methods, which could be remedied by milling sawtooth cuts into the surface of a thick multilayer using a focused-ion beam, for example.

We explored the applications of cut multilayers as a monochromator or spectrometer, a pulse compressor, and a volume zone plate. In the former case we considered multilayer structures that are cut with sawtooth profiles to extend their areas, and noted that other profiles can be cut into thick multilayer stacks to sensitively correct for phase aberrations, for example. The highest dispersion is achieved in the Laue or Bragg condensing geometries where the reflected wave approaches an angle parallel to the cut surface. This leads to compact designs of grating pulse compressors for positively chirped x-ray pulses from an FEL source (that is, pulses with longer wavelengths leading the pulse). For hard x rays of $1.5 \AA$ wavelength and shorter, we find the most compact and efficient designs are achieved in the Laue geometry. For soft $\mathrm{x}$ rays and EUV, these designs are usually too small, and reflective gratings provide a more convenient design. Finally, we calculate the local diffraction efficiency of hard x-ray volume zone plates and find that the optimum zone-plate profile is close to constant thickness.

\section{ACKNOWLEDGMENTS}

Preliminary investigations of cut multilayers were performed when SB and HNC were at Lawrence Livermore National Laboratory. We would like to thank Jennifer Alameda and Sherry Baker (LLNL) for technical help. The Advanced Light Source is supported by the Director, Office of Science, Office of Basic Energy Sciences, of the U.S. Department of Energy under contract no. DE-AC02-05CH11231.

\section{REFERENCES}

1. E. Spiller, "Low-loss reflection coatings using absorbing materials," Appl. Phys. Lett. 20, 365-367 (1972).

2. T. W. Barbee, "Combined microstructure x-ray optics," Rev. Sci. Instrum. 60, 1588-1595 (1989).

3. D. Hambach, G. Schneider, and E. M. Gullikson, "Efficient highorder diffraction of extreme-ultraviolet light and soft x-rays by nanostructured volume gratings," Opt. Lett. 26, 1200-1202 (2001).

4. J. C. Rife, W. R. Hunter, J. Troy, W. Barbee, and R. G. Cruddace, "Multilayer-coated blazed grating performance in the soft x-ray region," Appl. Opt. 28, 2984-2986 (1989).

5. D. L. Voronov, R. Cambie, E. M. Gullikson, V. V. Yashchuk, H. A. Padmore, Y. P. Pershin, A. G. Ponomarenko, and V. V. Kondratenko, "Fabrication and characterization of a new high density $\mathrm{Sc} / \mathrm{Si}$ multilayer sliced grating," Proc. SPIE 7077, 707708 (2008)
6. D. L. Voronov, M. Ahn, E. H. Anderson, R. Cambie, C.-H. Chang, E. M. Gullikson, R. K. Heilmann, F. Salmassi, M. L. Schattenburg, T. Warwick, V. V. Yashchuk, L. Zipp, and H. A. Padmore, "Highefficiency 5000 lines/mm multilayer-coated blazed grating for extreme ultraviolet wavelengths," Opt. Lett. 35, 2615-2617 (2010).

7. P. P. Naulleau, E. H. Anderson, E. M. Gullikson, and J. Bokor, "Fabrication of high-efficiency multilayer-coated binary blazed gratings in the EUV regime," Opt. Commun. 200, 27-34 (2001).

8. V. E. Levashov and A. V. Vinogradov, "Resonance diffraction efficiency enhancement in sliced multilayers," Appl. Opt. 32, 1130-1135 (1993).

9. V. Levashov, E. Zubarev, A. Fedorenko, V. Kondratenko, O. Poltseva, S. Yulin, I. Struk, and A. Vinogradov, "High throughput and resolution compact spectrograph for the 124-250 A range based on $\mathrm{MoSi}_{2}-\mathrm{Si}$ sliced multilayer grating," Opt. Commun. 109, 1-4 (1994).

10. M. Sánchez del Río and F. Cerrina, "Asymmetrically cut crystals for synchrotron radiation monochromators," Rev. Sci. Instrum. 63, 936-940 (1992).

11. S. Brauer, G. B. Stephenson, and M. Sutton, "Perfect crystals in the asymmetric Bragg geometry as optical elements for coherent x-ray beams," J. Synchrotron Rad. 2, 163-173 (1995).

12. A. Authier, Dynamical Theory of X-Ray Diffraction (Oxford University, 2001).

13. B. W. Batterman and H. Cole, "Dynamical diffraction of $x$ rays by perfect crystals," Rev. Mod. Phys. 36, 681-717 (1964).

14. J. Maser and G. Schmahl, "Coupled wave description of the diffraction by zone plates with high aspect ratios," Opt. Commun. 89, 355-362 (1992).

15. G. Schneider, "Zone plates with high efficiency in high orders of diffraction described by dynamical theory," Appl. Phys. Lett. 71, 2242-2244 (1997).

16. D. G. Stearns, "The scattering of $\mathrm{x}$ rays from nonideal multilayer structures,” J. Appl. Phys. 65, 491-506 (1989).

17. O. S. Heavens, Optical Properties of Thin Solid Films (Dover, 1965).

18. V. F. Sears, "Dynamical diffraction in periodic multilayers," Acta Cryst. A 53, 649-662 (1997).

19. M. Born and E. Wolf, Principles of Optics, 7th ed. (Cambridge University, 2002)

20. D. L. Windt, "IMD-software for modeling the optical properties of multilayer films," Comput. Phys. 12, 360-370 (1998).

21. B. L. Henke, E. M. Gullikson, and J. C. Davis, "X-ray interactions: photoabsorption, scattering, transmission, and reflection at $E=50-30000$ eV,$Z=1-92$," At. Data Nucl. Data Tables 54, 181-342 (1993).

22. X. Huang and M. Dudley, "A universal computation method for two-beam dynamical x-ray diffraction," Acta Cryst. A 59, 163-167 (2003)

23. L. De Caro and L. Tapfer, "Generalized Laue dynamical theory for x-ray reflectivity at low and high incidence angles on ideal crystals of finite size," Phys. Rev. B 55, 105-112 (1997).

24. S. Bajt, R. D. Behymer, P. B. Mirkarimi, C. Montcalm, M. A. Wall, M. Wedowski, and J. A. Folta, "Experimental investigation of beryllium-based multilayer coatings for extreme ultraviolet lithography," Proc. SPIE 3767, 259-270 (1999).

25. S. Bajt, D. G. Stearns, and P. A. Kearney, "Investigation of the amorphous-to-crystalline transition in Mo/Si multilayers," J. Appl. Phys. 90, 1017-1025 (2001).

26. H. N. Chapman, A. K. Ray-Chaudhuri, D. A. Tichenor, W. C. Replogle, R. H. Stulen, G. D. Kubiak, P. D. Rockett, L. E Klebanoff, D. O'Connell, A. H. Leung, K. L. Jefferson, J. B Wronosky, J. S. Taylor, L. C. Hale, K. Blaedel, E. A. Spiller, G. E. Sommargren, J. A. Folta, D. W. Sweeney, E. M. Gullikson, P. Naulleau, K. A. Goldberg, J. Bokor, D. T. Attwood, U. Mickan, R. Hanzen, E. Panning, P.-Y. Yan, C. W. Gwyn, and S. H. Lee, "First lithographic results from the extreme ultraviolet Engineering Test Stand," J. Vac. Sci. Technol. B 19, 2389-2395 (2001).

27. E. M. Gullikson, S. Mrowka, and B. B. Kaufmann, "Recent developments in EUV reflectometry at the Advanced Light Source," Proc. SPIE 4343, 363-373 (2001).

28. D. G. Stearns, M. B. Stearns, Y. Cheng, J. H. Stith, and N. M. Ceglio, "Thermally induced structural modification of Mo-Si multilayers," J. Appl. Phys. 67, 2415-2427 (1990). 
29. S. Bajt, J. Alameda, J. T. Barbee, W. M. Clift, J. A. Folta, B. Kaufmann, and E. Spiller, "Improved reflectance and stability of Mo/Si mutilayers," Opt. Eng. 41, 1797-1804 (2002).

30. S. Braun, H. Mai, M. Moss, R. Scholz, and A. Leson, "Mo/Si multilayers with different barrier layers for applications as extreme ultraviolet mirrors," Jpn. J. Appl. Phys. 41, 4074-4081 (2002).

31. S. Bajt and D. G. Stearns, "High temperature stability multilayers for extreme ultraviolet condenser optics," Appl. Opt. 44, 77357743 (2005).

32. H. N. Chapman and J. S. Taylor, "Etched-multilayer phase shifting masks for EUV lithography," U.S. patent 6,875,543 B2 (April 5 2005).

33. A. G. Michette, Optical Systems for Soft X Rays (Plenum, 1986).

34. V. V. Aristov, A. I. Erko, and V. V. Martynov, "Principles of BraggFresnel multilayer optics," Rev. Phys. Appl. 23, 1623-1630 (1988).

35. A. I. Erko, V. V. Aristov, and B. Vidal, Diffraction X-Ray Optics (IOP, 1996).

36. V. V. Aristov, A. A. Snigirev, Yu. A. Basov, and A. Yu. Nikulin, "Xray Bragg optics," AIP Conf. Proc. 147, 253-259 (1986).

37. C. Pellegrini, "High power femtosecond pulses from an x-ray SASE-FEL," Nucl. Instrum. Meth. Phys. Res. A 445, 124-127 (2000).

38. H. Chapman and K. Nugent, "X-ray pulse compression using strained crystals," Opt. Commun. 205, 351-359 (2002).

39. P. Emma, R. Akre, J. Arthur, R. Bionta, C. Bostedt, J. Bozek, A. Brachmann, P. Bucksbaum, R. Coffee, F. J. Decker, Y. Ding, D. Dowell, S. Edstrom, A. Fisher, J. Frisch, S. Gilevich, J. Hastings, G. Hays, P. Hering, Z. Huang, R. Iverson, H. Loos, M. Messerschmidt, A. Miahnahri, S. Moeller, H. D. Nuhn, G. Pile, D. Ratner, J. Rzepiela, D. Schultz, T. Smith, P. Stefan, H. Tompkins, J. Turner, J. Welch, W. White, J. Wu, G. Yocky, and J. Galayda, "First lasing and operation of an ångstromwavelength free-electron laser," Nat. Photon. 4, 641-647 (2010).
40. J. Arthur, "LCLS design study report," Internal Technical Report 521, SLAC (Stanford University, 1998).

41. J. Kirz, C. Jacobsen, and M. Howells, "Soft x-ray microscopes and their biological applications," Q. Rev. Biophys. 28, 33130 (1995).

42. W. Chao, B. D. Harteneck, J. A. Liddle, E. H. Anderson, and D. T. Attwood, "Soft x-ray microscopy at a spatial resolution better than 15 nm," Nature 435, 1210-1213 (2005).

43. H. C. Kang, G. B. Stephenson, C. Liu, R. Conley, A. T. Macrander, J. Maser, S. Bajt, and H. N. Chapman, "High-efficiency diffractive x-ray optics from sectioned multilayers," Appl. Phys. Lett. 86, 151109 (2005).

44. H. C. Kang, J. Maser, G. B. Stephenson, C. Liu, R. Conley, A. T. Macrander, and S. Vogt, "Nanometer linear focusing of hard $\mathrm{x}$ rays by a multilayer Laue lens," Phys. Rev. Lett. 96, 127401 (2006).

45. H. C. Kang, H. Yan, R. P. Winarski, M. V. Holt, J. Maser, C. Liu, R. Conley, S. Vogt, A. T. Macrander, and G. B. Stephenson, "Focusing of hard x-rays to 16 nanometers with a multilayer Laue lens," Appl. Phys. Lett. 92, 221114 (2008).

46. D. Rudolph and G. Schmahl, "High power zone plates for a soft x-ray microscope," Ann. N.Y. Acad. Sci. 342, 94-104 (1980).

47. F. Pfeiffer, C. David, J. F. van der Veen, and C. Bergemann, "Nanometer focusing properties of Fresnel zone plates described by dynamical diffraction theory," Phys. Rev. B 73, 245331 (2006).

48. L. Solymar and D. J. Cooke, Volume Holography and Volume Gratings (Academic, 1981)

49. H. Yan, J. Maser, A. Macrander, Q. Shen, S. Vogt, G. B. Stephenson, and H. C. Kang, "Takagi-Taupin description of $\mathrm{x}$ ray dynamical diffraction from diffractive optics with large numerical aperture," Phys. Rev. B 76, 115438 (2007). 